\title{
Inborn errors of type I IFN immunity in patients with life-threatening COVID-19
}

Qian Zhang', Paul Bastard ${ }^{2,3 *}$, Zhiyong Liu' ${ }^{1 *}$, Jérémie Le Pen ${ }^{4 *}$, Marcela Moncada-Velez ${ }^{1 *}$, Jie Chen ${ }^{1 *}$, Masato Ogishi $^{1 *}$, Ira K. D. Sabli5*, Stephanie Hodeib ${ }^{5 *}$, Cecilia Korol ${ }^{2 *}$, Jérémie Rosain ${ }^{2,3 *}$, Kaya Bilguvar6*, Junqiang Ye $^{7 *}$, Alexandre Bolze ${ }^{8 *}$, Benedetta Bigio ${ }^{1 *}$, Rui Yang'*, Andrés Augusto Arias ${ }^{1,9,10 *}$, Qinhua Zhou ${ }^{1 *}$, Yu $^{2,3}$ Zhang'11,12*, Fanny Onodi ${ }^{13}$, Sarantis Korniotis ${ }^{13}$, Léa Karpf ${ }^{13}$, Quentin Philippot ${ }^{2,3}$, Marwa Chbihi ${ }^{2,3}$, Lucie Bonnet-Madin $^{14}$, Karim Dorgham ${ }^{15}$, Nikaïa Smith ${ }^{16}$, William M. Schneider ${ }^{4}$, Brandon S. Razooky ${ }^{4}$, HansHeinrich Hoffmann ${ }^{4}$, Eleftherios Michailidis ${ }^{4}$, Leen Moens ${ }^{17}$, Ji Eun Han ${ }^{1}$, Lazaro Lorenzo ${ }^{2,3}$, Lucy Bizien $^{2,3}$, Philip Meade ${ }^{18}$, Anna-Lena Neehus ${ }^{2,3}$, Aileen Camille Ugurbil', Aurélien Corneau ${ }^{19}$, Gaspard Kerner ${ }^{2,3}$, Peng Zhang', Franck Rapaport $^{1}$, Yoann Seeleuthner ${ }^{2,3}$, Jeremy Manry $^{2,3}$, Cecile Masson ${ }^{20}$, Yohann Schmitt ${ }^{20}$, Agatha Schlüter $^{21}$, Tom Le Voyer ${ }^{2,3}$, Taushif Khan ${ }^{22}$, Juan Li ${ }^{1}$, Jacques Fellay ${ }^{23,24,25}$, Lucie Roussel ${ }^{26}$, Mohammad Shahrooei ${ }^{27,28}$, Mohammed F. Alosaimi ${ }^{29}$, Davood Mansouri ${ }^{30,31,32}$, Haya Al-Saud ${ }^{33}$, Fahd Al-Mulla ${ }^{34}$, Feras Almourfi $^{33}$, Saleh Zaid Al-Muhsen ${ }^{35}$, Fahad Alsohime ${ }^{29}$, Saeed Al Turki36,37, Rana Hasanato ${ }^{29}$, Diederik van de Beek $^{38}$, Andrea Biondi ${ }^{39}$, Laura Rachele Bettini ${ }^{39}$, Mariella D'Angio ${ }^{39}$, Paolo Bonfanti ${ }^{40}$, Luisa Imberti ${ }^{41}$,

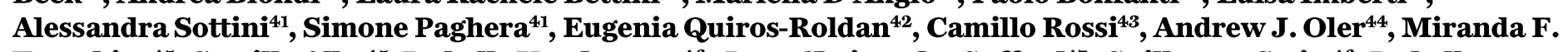
Tompkins $^{45}$, Camille Alba ${ }^{45}$, Isabelle Vandernoot ${ }^{46}$, Jean-Christophe Goffard ${ }^{47}$, Guillaume Smits ${ }^{46}$, Isabelle Migeotte $^{48}$, Filomeen Haerynck ${ }^{49}$, Pere Soler-Palacin ${ }^{50}$, Andrea Martin-Nalda ${ }^{50}$, Roger Colobran ${ }^{51}$, PierreEmmanuel Morange ${ }^{52}$, Sevgi Keles ${ }^{53}$, Fatma Çölkesen ${ }^{54}$, Tayfun Ozcelik ${ }^{55}$, Kadriye Kart Yasar ${ }^{56}$, Sevtap Senoglu $^{56}$, Şemsi Nur Karabela ${ }^{56}$, Carlos Rodríguez Gallego ${ }^{57,58}$, Giuseppe Novelli59, Sami Hraiech ${ }^{60}$, Yacine Tandjaoui-Lambiotte $^{61,62}$, Xavier Duval ${ }^{63,64}$, Cédric Laouénan ${ }^{63,64,65}$, COVID-STORM Clinicians†, COVID Clinicians $\dagger$, Imagine COVID Group $\dagger$, French COVID Cohort Study Group $\dagger$, CoV-Contact Cohort + , Amsterdam UMC Covid-19 Biobank + , COVID Human Genetic Effort + , NIAID-USUHS/TAGC COVID Immunity Group $\dagger$, Andrew L. Snow ${ }^{66}$, Clifton L. Dalgard ${ }^{45,67}$, Joshua Milner ${ }^{68}$, Donald C. Vinh ${ }^{26}$, Trine H. Mogensen ${ }^{69,70}$, Nico Marr $^{22,71}$, András N. Spaan ${ }^{1,72}$, Bertrand Boisson ${ }^{1,2,3}$, Stéphanie Boisson-Dupuis ${ }^{1,2,3}$, Jacinta Bustamante ${ }^{1,2,3,73}$, Anne Puel ${ }^{1,2,3}$, Michael Ciancanelli ${ }^{1,74}$, Isabelle Meyts ${ }^{17,75}$, Tom Maniatis ${ }^{7,76}$, Vassili Soumelis ${ }^{13,77}$, Ali Amara ${ }^{14}$, Michel Nussenzweig ${ }^{78,79}$, Adolfo García-Sastre ${ }^{18,80,81,82}$, Florian Krammer ${ }^{18}$, Aurora Pujol ${ }^{21}$, Darragh Duffy ${ }^{16}$, Richard Lifton $^{83,84,85}$, , Shen-Ying Zhang ${ }^{1,2,3}$, Guy Gorochov ${ }^{15}$, , Vivien Béziat ${ }^{1,2,3}$, Emmanuelle Jouanguy ${ }^{1,2,3 \ddagger}$ Vanessa Sancho-Shimizu ${ }^{5}$, Charles M. Rice ${ }^{4}$, Laurent Abel ${ }^{1,2,3}$ \&, Luigi D. Notarangelo ${ }^{11,12} \S$, Aurélie Cobat $^{1,2,3} \S$, Helen C. Su ${ }^{11,12} \S$, Jean-Laurent Casanova ${ }^{1,2,3,79,86} \S$

${ }^{1}$ St. Giles Laboratory of Human Genetics of Infectious Diseases, Rockefeller Branch, The Rockefeller University, New York, NY, USA. ${ }^{2}$ Laboratory of Human Genetics of Infectious Diseases, Necker Branch, INSERM U1163, Necker Hospital for Sick Children, Paris, France. ${ }^{3}$ University of Paris, Imagine Institute, Paris, France. ${ }^{4}$ Laboratory of Virology and Infectious Disease, The Rockefeller University, New York, NY, USA. ${ }^{5}$ Department of Paediatric Infectious Diseases \& Virology, Imperial College London, Norfolk Place, London, UK. ${ }^{6}$ Yale Center for Genome Analysis and Department of Genetics, Yale School of Medicine, New Haven, CT, USA. ${ }^{7}$ Zukerman Mind Brain Behavior Institute, Columbia University, New York, NY, USA. ${ }^{8}$ Helix, San Mateo, CA, USA. ${ }^{9}$ Primary Immunodeficiencies Group, University of Antioquia UdeA, Medellin, Colombia. ${ }^{10}$ School of Microbiology, University of Antioquia UdeA, Medellin, Colombia. ${ }^{11}$ Laboratory of Clinical Immunology and Microbiology, Division of Intramural Research, NIAID, NIH, Bethesda, MD, USA. ${ }^{12}$ NIAID Clinical Genomics Program, National Institutes of Health, Bethesda, MD, USA. ${ }^{13}$ Université de Paris, Institut de Recherche Saint-Louis, INSERM U976, Hôpital Saint-Louis, 75010 Paris, France. ${ }^{14}$ Laboratory of Genomes \& Cell Biology of Disease, INSERM U944, CNRS UMR 7212, Université de Paris, Institut de Recherche Saint-Louis, Hôpital Saint-Louis, Paris, France. ${ }^{15}$ Sorbonne Université, Inserm, Centre d'immunologie et des maladies infectieuses-Paris (CIMI PARIS), AP-HP Hôpital Pitié-Salpêtrière, Paris, France. ${ }^{16}$ Translational Immunology Lab, Institut Pasteur, Paris, France. ${ }^{17}$ Laboratory for Inborn Errors of Immunity, Department of Microbiology, Immunology and Transplantation, Department of Pediatrics, University Hospitals Leuven, KU Leuven, Belgium. ${ }^{18}$ Department of Microbiology, Icahn School of Medicine at Mount Sinai, New York, NY, USA. ${ }^{19}$ Sorbonne Université, UMS037, PASS, Plateforme de cytométrie de la Pitié-Salpêtrière CyPS, F-75013 Paris, France. ${ }^{20}$ Bioinformatics Platform, Structure Fédérative de Recherche Necker, INSERM UMR1163, Université de Paris, Imagine Institute, Paris, France. ${ }^{21}$ Neurometabolic Diseases Laboratory, IDIBELL-Hospital Duran i Reynals, CIBERER U759, and Catalan Institution of Research and Advanced Studies (ICREA), Barcelona, Spain. ${ }^{22}$ Department of Immunology, Research Branch, Sidra Medicine, Doha, Qatar. ${ }^{23}$ School of Life sciences, Ecole Polytechnique Fédérale de Lausanne, Lausanne, Switzerland. ${ }^{24}$ Precision Medicine Unit, Lausanne University Hospital and University of Lausanne, Lausanne, Switzerland. ${ }^{25}$ Swiss Institue of Bioinformatics, Lausanne, Switzerland. ${ }^{26}$ Infectious Disease Susceptibility Program, Research Institute-McGill University Health Centre, Montréal, Québec, Canada. ${ }^{27}$ Specialized Immunology Laboratory of Dr. Shahrooei, Sina Medical Complex, Ahvaz, Iran. ${ }^{28}$ Department of Microbiology and Immunology, Clinical and Diagnostic Immunology, KU Leuven, Leuven, Belgium. ${ }^{29}$ Department of Pediatrics, College of Medicine, King Saud University, Riyadh, Saudi Arabia. ${ }^{30}$ Department of Clinical Immunology and Infectious Diseases, National Research Institute of Tuberculosis and Lung Diseases, Shahid Beheshti University of Medical Sciences, Tehran, Iran. ${ }^{31}$ The Clinical Tuberculosis and Epidemiology Research Center, National Research Institute of, Tuberculosis and Lung Diseases (NRITLD), Masih Daneshvari Hospital, Shahid Beheshti, University of Medical Sciences, Tehran, Iran. 
${ }^{32}$ Pediatric Respiratory Diseases Research Center, National Research Institute of Tuberculosis and Lung Diseases, Shahid Beheshti, Iran. ${ }^{33}$ National Center of Genomics Technology, King Abdulaziz City for Science and Technology, Riyadh, Saudi Arabia. ${ }^{34}$ Dasman Diabetes Institute, Department of Genetics and Bioinformatics, Kuwait. ${ }^{35}$ Immunology Research Laboratory, Department of Pediatrics, College of Medicine and King Saud University Medical City, King Saud University, Riyadh, Saudi Arabia. ${ }^{36}$ Translational Pathology, Department of Pathology and Laboratory Medicine, King Abdulaziz Medical City, Misery of National Guard- Health Affairs, Riyadh, Saudi Arabia. ${ }^{37}$ Cancer \& Blood Research, King Abdullah International Medical Research Center, Riyadh, KSA. ${ }^{38}$ Amsterdam UMC, Department of Neurology, Amsterdam Neuroscience, Amsterdam, Netherlands. ${ }^{39}$ Pediatric Departement and Centro Tettamanti-European Reference Network PaedCan, EuroBloodNet, MetabERN-University of Milano-BicoccaFondazione MBBM-Ospedale, San Gerardo, Monza, Italy. ${ }^{40}$ Department of Infectious Diseases, San Gerardo Hospital - University of Milano-Bicocca, Monza, Italy. ${ }^{41} \mathrm{CREA}$ Laboratory, Diagnostic Laboratory, ASST Spedali Civili di Brescia, Brescia, Italy. ${ }^{42}$ Department of Infectious and Tropical Diseases, University of Brescia and ASST Spedali di Brescia, Brescia, Italy. ${ }^{43}$ Chief Medical Officer, ASST Spedali Civili di Brescia, Brescia, Italy. ${ }^{44}$ Bioinformatics and Computational Biosciences Branch, Office of Cyber Infrastructure and Computational Biology, NIAID, NIH, Bethesda, MD, USA. ${ }^{45}$ PRIMER, Uniformed Services University of the Health Sciences, Bethesda, MD, USA. ${ }^{46}$ Center of Human Genetics, Hôpital Erasme, Université Libre de Bruxelles, Brussels, Belgium. ${ }^{77}$ Department of Internal Medicine, Hôpital Erasme, Université Libre de Bruxelles, Brussels, Belgium. ${ }^{48}$ Fonds de la Recherche Scientifique (FNRS) and Center of Human Genetics, Hôpital Erasme, Université Libre de Bruxelles, Brussels, Belgium. ${ }^{49}$ Department of Paediatric Immunology and Pulmonology, Centre for Primary Immunodeficiency Ghent (CPIG), PID research lab, Jeffrey Modell Diagnosis and Research Centre, Ghent University Hospital, Ghent, Belgium. ${ }^{50}$ Pediatric Infectious Diseases and Immunodeficiencies Unit, Hospital Universitari Vall d'Hebron, Vall d'Hebron Research Institute, Vall d'Hebron Barcelona Hospital Campus. Universitat Autònoma de Barcelona (UAB). Barcelona, Catalonia, Spain. ${ }^{51}$ Immunology Division, Genetics Department. Hospital Universitari Vall d'Hebron. Vall d'Hebron Research Institute. Vall d'Hebron Barcelona Hospital Campus. Universitat Autònoma de Barcelona (UAB). Barcelona, Catalonia, Spain. ${ }^{52}$ Aix Marseille Univ, INSERM, INRAE, C2VN, CHU Timone, Marseille, France. ${ }^{53}$ Necmettin Erbakan University, Meram Medical Faculty, Division of Pediatric Allergy and Immunology, Konya, Turkey ${ }^{54}$ Department of Infectious Diseases and Clinical Microbiology, Konya Training and Research Hospital, Konya, Turkey. ${ }^{55}$ Bilkent University, Department of Molecular Biology and Genetics, Bilkent - Ankara 06800, Turkey. ${ }^{56}$ Departments of Infectious Diseases and Clinical Microbiology, Bakirkoy Dr. Sadi Konuk Training and Research Hospital, University of Health Sciences, Istanbul, Turkey. ${ }^{57}$ Department of Immunology, Hospital Universitario de G.C. Dr. Negrín, Canarian Health System, Las Palmas de Gran Canaria, Spain. ${ }^{58}$ University Fernando Pessoa Canarias, Las Palmas de Gran Canaria, Spain. ${ }^{59}$ Department of Biomedicine and Prevention, University of Rome "Tor Vergata", Rome, Italy. ${ }^{60}$ Intensive Care Unit, AP-HM, Marseille, France. ${ }^{61}$ Avicenne Hospital Intensive Care Unit, APHP, Bobigny, INSERM U1272 Hypoxia \& Lung, Paris, France. ${ }^{62}$ PH Réanimation CHU Avicenne, Bobigny, INSERM U1272 Hypoxie \& Poumon, Paris, France. ${ }^{63}$ Université de Paris, IAME UMR-S 1137 , INSERM, Paris, France, EU. ${ }^{64}$ Inserm CIC 1425, Paris, France. ${ }^{65} \mathrm{AP}$-HP, Département Epidémiologie Biostatistiques et Recherche Clinique, Hôpital Bichat, Paris, France, EU. ${ }^{66}$ Department of Pharmacology \& Molecular Therapeutics, Uniformed Services University of the Health Sciences, Bethesda, MD, USA. ${ }^{67}$ Department of Anatomy, Physiology \& Genetics, Uniformed Services University of the Health Sciences, Bethesda, MD, USA. ${ }^{68}$ Division of Pediatric Allergy, Immunology and Rheumatology, Columbia University, New York, USA. ${ }^{69}$ Department of Infectious Diseases, Aarhus University Hospital, Skejby, Denmark. ${ }^{70}$ Department of Biomedicine, Aarhus University, Denmark. ${ }^{71} \mathrm{College}$ of Health and Life Sciences, Hamad Bin Khalifa University, Doha, Qatar. ${ }^{72}$ Department of Medical Microbiology, University Medical Center Utrecht, Utrecht, Netherlands. ${ }^{73}$ Study Center for Primary Immunodeficiencies, Necker Hospital for Sick Children, 75015 Paris, France. ${ }^{74}$ Turnstone Biologics, New York, NY, USA. ${ }^{75}$ Department of Pediatrics, University Hospitals Leuven, KU Leuven, Belgium. ${ }^{76}$ New York Genome Center, New York, NY, USA. ${ }^{77}$ Assistance Publique-Hôpitaux de Paris (AP-HP), Hôpital Saint-Louis, Laboratoire d'Immunologie, Paris, France. ${ }^{78}$ Laboratory of Molecular Immunology, Rockefeller University, New York, NY, USA. ${ }^{79} \mathrm{Howard}$ Hughes Medical Institute, New York, NY, USA. ${ }^{80}$ Department of Medicine, Division of Infectiosu Diseases, Icahn School of Medicine at Mount Sinai, New York, NY, USA. ${ }^{81}$ Global Health and Emerging Pathogens Institute, Icahn School of Medicine at Mount Sinai, New York, NY, USA. ${ }^{82}$ The Tisch Cancer Institute, Icahn School of Medicine at Mount Sinai, New York, NY, USA. ${ }^{83}$ Laboratory of Genetics and Genomics, The Rockefeller University, New York, NY, USA. ${ }^{84}$ Department of Genetics, Yale University School of Medicine, New Haven, CT, USA. ${ }^{85}$ Yale Center for Genome Analysis, Yale School of Medicine, New Haven, CT, USA. ${ }^{86}$ Pediatric Hematology and Immunology Unit, Necker Hospital for Sick Children, AP-HP, Paris, France.

*These authors contributed equally to this work.

†All collaborators and their affiliations appear at the end of this paper.

$\ddagger$ These authors contributed equally to this work.

§These authors contributed equally to this work.

ๆCorresponding author. Email: casanova@rockefeller.edu

Clinical outcome upon infection with SARS-CoV-2 ranges from silent infection to lethal COVID-19. We have found an enrichment in rare variants predicted to be loss-of-function (LOF) at the 13 human loci known to govern TLR3- and IRF7-dependent type I interferon (IFN) immunity to influenza virus, in 659 patients with life-threatening COVID-19 pneumonia, relative to 534 subjects with asymptomatic or benign infection. By testing these and other rare variants at these 13 loci, we experimentally define LOF variants in 23 patients (3.5\%), aged 17 to 77 years, underlying autosomal recessive or dominant deficiencies. We show that human fibroblasts with mutations affecting this pathway are vulnerable to SARS-CoV-2. Inborn errors of TLR3- and IRF7-dependent type I IFN immunity can underlie life-threatening COVID-19 pneumonia in patients with no prior severe infection. 
SARS-CoV-2 has already claimed at least 800,000 lives, has been detected in at least 20 million people, and has probably infected at least another 200 million. The clinical manifestations range from silent infection to lethal disease, with an infection-fatality rate of $0.1 \%$ to $0.9 \%$. Three epidemiological factors increase the risk of severity: increasing age, decadeby-decade, after the age of 50 years, being male and various underlying medical conditions (1). However, even taking these factors into account, there is immense interindividual clinical variability in each demographic category considered. Following on from our human genetic studies of other severe infectious diseases $(2,3)$, we established the COVID Human Genetic Effort (https://www.covidhge.com) to test the general hypothesis that life-threatening COVID-19 may be caused, in some patients, by monogenic inborn errors of immunity to SARS-CoV-2, with incomplete or complete penetrance (4). We enrolled 659 patients $(74.5 \%$ men and $25.5 \%$ women, $13.9 \%$ of whom died) of various ancestries, aged between one month and 99 years (Fig. 1A). These patients were hospitalized for life-threatening pneumonia due to SARSCoV-2 (critical COVID-19). We sequenced their whole genome $(N=364)$ or exome $(N=295)$, and principal component analysis (PCA) on these data confirmed their ancestries (Fig. 1B).

\section{Candidate variants at thirteen human loci that govern immunity to influenza virus}

We first tested the specific hypothesis that inborn errors of TLR3- and IRF7-dependent type I interferon (IFN) immunity, which underlie life-threatening influenza pneumonia, may also underlie life-threatening COVID-19 pneumonia (5) (Fig. 2). We considered three loci previously shown to be mutated in patients with critical influenza pneumonia: TLR3 (6), IRF7 (7), and IRF9 (8). We also considered 10 loci mutated in patients with other viral illnesses but directly connected to the three core genes conferring influenza susceptibility: TICAMI/TRIF (9), UNC93B1 (10), TRAF3 (11), TBK1 (12), IRF3 (13) and NEMO/IKBKG (14) in the TLR3-dependent type I IFN induction pathway, and IFNARI (15), IFNAR2 (16), STAT1 (17), and STAT2 (18) in the IRF7- and IRF9dependent type I IFN amplification pathway. We collected both mono- and biallelic non-synonymous variants with a minor allele frequency $(\mathrm{MAF})<0.001$ at all 13 loci. Twelve of the thirteen candidate loci are autosomal, while NEMO is X-linked. For the latter gene, we considered only a recessive model (19). Autosomal dominant (AD) inheritance has not been proven for six of the 12 autosomal loci (UNC93B1, IRF7, IFNAR1, IFNAR2, STAT2, IRF9), but we nevertheless considered heterozygous variants, because none of the patients enrolled had been hospitalized for critical viral infections before COVID-19, raising the possibility that any underlying genetic defects they might have display a lower penetrance for influenza and other viral illnesses than for COVID-19, which is triggered by a more virulent virus.

\section{Enrichment of variants predicted to be loss-of-function at the influenza susceptibility loci}

We found four unrelated patients with biallelic variants of IRF7 or IFNAR1 (Table 1 and table S1). We also found 113 patients carrying 113 monoallelic variants at 12 loci: TLR3 $(N$ = 7 patients $/ 7$ variants), UNC93B1 $(N=10 / 9), \operatorname{TICAM1}(N=$ $17 / 15)$, TRAF3 $(N=6 / 6)$, TBKI $(N=12 / 11), I R F 3(N=5 / 5)$, IRF7 $(N=20 / 13), \operatorname{IFNARI}(N=14 / 13), \operatorname{IFNAR2}(N=17 / 15)$, $\operatorname{STAT1}(N=4 / 4), \operatorname{STAT2}(N=11 / 11)$, and IRF9 $(N=4 / 4)$. We detected no copy number variation (CNV) for these 13 genes. Remarkably, one of these variants has been reported in patients with life-threatening influenza pneumonia (TLR3 p.Pro554Ser) $(6,20)$, and another was shown to be both deleterious and dominant-negative (IFNAR1 p.Pro335del) (21). Nine of the 118 biallelic or monoallelic variants were predicted to be loss-of-function (pLOF), whereas the remaining 109 were missense or in-frame indels (table S1). In a sample of 534 controls with asymptomatic or mild SARS-CoV-2 infection, we found only one heterozygous pLOF variation with a MAF $<0.001$ at the 13 loci (IRF7 p.Leu99fs). A PCA-adjusted burden test on the 12 autosomal loci revealed significant enrichment in pLOF variants in patients relative to controls ( $p$ $=0.01, \mathrm{OR}=8.28[1.04-65.64,95 \% \mathrm{CI}])$ under an $\mathrm{AD}$ mode of inheritance. The same analysis performed on synonymous variants with a MAF $<0.001$ was not significant $(p=0.19$ ), indicating that our ethnicity-adjusted burden test was well calibrated.

\section{Experimentally deleterious alleles at the influenza sus- ceptibility loci in $3.5 \%$ of the patients}

We tested 113 of these 118 variants experimentally in ad hoc overexpression systems. We found that 24 variants of eight genes were deleterious (including all the pLOF variants), as they were loss-of-expression (LOE), LOF, or severely hypomorphic (HYPO): TLR3 ( $N=4$ variants), UNC93BI $(N=1)$, TICAM1 $(N=3), \operatorname{TBK1}(N=2), \operatorname{IRF3}(N=2), \operatorname{IRF} 7(N=8)$, $\operatorname{IFNARI}(N=3)$, and IFNAR2 $(N=1)$ (table S1, Fig. 3, and figs. S1 to S8). Consistently, heterozygous LOF variants of IRF3 and $I R F 7$ were reported in single patients with life-threatening influenza pneumonia $(22,23)$. The remaining 89 variants tested were biochemically neutral. Twenty-three patients carried these 24 deleterious variants, resulting in four autosomal recessive (AR) deficiencies (homozygosity or compound heterozygosity for $I R F 7$, homozygosity for IFNARI) and $19 \mathrm{AD}$ deficiencies. These 23 patients did not carry candidate variants at the other 417 loci known to underlie inborn errors of immunity (table S2) (24-26). These findings suggest that at least $23(3.5 \%)$ unrelated patients of the 659 patients tested suffered from a deficiency at one of eight loci among the 13 tested: four patients with a known $\mathrm{AR}$ disorder (IRF7, IFNARI) $(7,15)$, eleven with a known $\mathrm{AD}$ disorder (TLR3, TICAM1, TBK1, IRF3) $(6,9,12,13,20)$, and eight with a previously unknown $\mathrm{AD}$ genetic disorder (UNC93B1, IRF7, IFNAR1, IFNAR2).

\section{Impaired TLR3- and IRF7-dependent type I immunity in the patients' cells in vitro}

We tested cells from patients with selected genotypes. We showed that PHA-driven T-cell blasts (PHA-T cells) from 
patients with AR or AD IRF7 deficiency had low levels of IRF7 expression (Fig. 4A). We then isolated circulating plasmacytoid dendritic cells (pDCs) from a patient with AR IRF7 deficiency (fig. S9A) (7). These cells were present in normal proportions (fig. S9B), but they did not produce any detectable type I or III IFNs in response to SARS-CoV-2, as analyzed by cytometric bead array (CBA), ELISA, and RNA-seq (Fig. 4, $\mathrm{B}$ and $\mathrm{C})$. We also showed that PHA-T cells from a patient with AR IFNAR1 deficiency had impaired IFNAR1 expression and responses to IFN- $\alpha 2$ or $-\beta$, and that the patient's SV40transformed fibroblast (SV40-Fib cells) did not respond to IFN- $\alpha 2$ and $-\beta$ (Fig. 5). We then infected $\mathrm{TLR}^{-/-} \mathrm{TLR}^{+/-}$, $\mathrm{IRF}^{-/-}, \mathrm{IRF} 7^{-/-}$rescued with wild-type IRF7, IFNAR1 ${ }^{-/-}$, and IFNAR1 $^{-1-}$ rescued with wild-type IFNAR1 SV40-Fib cells previously transduced with ACE2 and TMPRSS2. SARS-CoV-2 infection levels were higher in mutant cells than in cells from healthy donors, and transduction of wild-type $I R F 7$ or IFNARI rescued their defects (Fig. 6). Collectively, these findings showed that AR IRF7 deficiency impaired the production of type I IFN by pDCs stimulated with SARS-CoV-2, whereas $\mathrm{AR}$ and $\mathrm{AD}$ deficiencies of TLR3, or AR deficiency of IFNAR1 impaired fibroblast-intrinsic type I IFN immunity to SARSCoV2. They also suggest that heterozygosity for LOF variations at the other five mutated loci also underlie life-threatening COVID-19.

\section{Impaired production of type I IFNs in the patients in vivo}

We tested whether these genotypes impaired the production of type I IFN in vivo, during the course of SARS-CoV-2 infection. We measured the levels of the 13 types of IFN- $\alpha$ in the blood of patients during the acute phase of COVID-19. We found that 10 of the 23 patients with mutations for whom samples were available (one with AR IRF7 deficiency, four with AD IRF7 deficiency, one with AD TLR3 deficiency, two with AD TBK1 deficiency, one with AR IFNAR1 deficiency, and one with AD TICAM1 deficiency) had serum IFN- $\alpha$ levels below 1 pg/mL (Fig. 7). By contrast, previously published cohorts of patients hospitalized with unexplained, severe COVID-19 had various serum IFN- $\alpha$ levels, significantly higher than our 10 patients (one-way ANOVA, $p=$ 1.4x10 ${ }^{-7}$; Fig. 7) $(27,28)$. Importantly, another 29 patients from our cohort displaying auto-Abs against type I IFNs, reported in an accompanying paper, had undetectable levels of serum IFN- $\alpha$ (29). Moreover, none of the 23 patients with LOF mutations of the eight genes had detectable auto-Abs against type I IFNs (29), strongly suggesting that the two mechanisms of disease are similar, but independent. Strikingly, excluding patients with autoAbs against type I IFN from the burden test of pLOF variants at the 12 autosomal loci strengthened the association signal ( $p=$ $0.007, \mathrm{OR}=8.97[1.13-71.09,95 \% \mathrm{CI}])$.

\section{Inborn errors of TLR3- and IRF7-dependent type I im- munity underlie critical COVID-19}

Collectively, our data suggest that at least 23 of the 659 patients with life-threatening COVID-19 pneumonia studied have known (six disorders) or new (four disorders) genetic defects at eight loci involved in the TLR3- and IRF7dependent induction and amplification of type I IFNs. This discovery reveals the essential role of both the dsRNA sensor TLR3 and type I IFN cell-intrinsic immunity in the control of SARS-CoV-2 infection in the lungs, consistent with their previously documented roles in pulmonary immunity to influenza virus (5-8). Strikingly, these genotypes were silent until infection with SARS-CoV-2. The most thought-provoking examples are the AR deficiencies of IRF7 and IFNAR1. AR IRF7 deficiency was diagnosed in two individuals aged 49 and 50 years, and AR IFNAR1 deficiency was diagnosed in two individuals aged 26 and 38 years, with no prior history of lifethreatening infections (Table 1). One patient with IRF7 deficiency tested was seropositive for several common viruses, including various influenza A and B viruses (figs. S10 and S11). These genetic defects therefore display incomplete penetrance for influenza respiratory distress, and only manifested clinically upon infection with the more virulent SARS-CoV-2.

\section{Conclusion}

The AR form of IFNAR1 deficiency highlights the importance of type I IFN production, relative to type III IFN, the production of which is also impaired by defects of TLR3, IRF7, and IRF9 (5). This conclusion is also supported by our accompanying report of neutralizing auto-antibodies against type I, but not type III IFNs, in other patients with life-threatening COVID-19 pneumonia (29). Inborn errors of TLR3- and IRF7dependent type I IFN immunity at eight loci were found in as many as 23 patients (3.5\%) of various ages ( 17 to 77 years) and ancestries (various nationalities, from Asia, Europe, Latin America and the Middle East), and in patients of both sexes (Table 1). Our findings suggest that there may be mutations in other type I IFN-related genes in other patients with lifethreatening COVID-19 pneumonia. They also suggest that the administration of type I IFN may be of therapeutic benefit in selected patients, at least early in the course of SARS-CoV-2 infection.

\section{Methods \\ Patients}

We included in this study 659 patients with life-threatening COVID-19 pneumonia defined as patients with pneumonia who developed critical disease, whether pulmonary with mechanical ventilation (CPAP, BIPAP, intubation, hi-flow oxygen), septic shock, or with any other organ damage requiring admission to the ICU. Patients who developed Kawasaki-like syndrome were excluded. The age of the patients ranged from 0.1-99 years, with a mean age of 51.8 years (SD 15.9 years), and $25.5 \%$ of the patients were female. As controls, we enrolled 534 individuals infected with SARS-CoV-2 (based on a positive PCR and/or serological test and/or the presence of typical symptoms such as anosmia/ageusia after exposure to a confirmed COVID-19 case) who remained asymptomatic or developed mild, self-healing, ambulatory disease. 


\section{Next-generation sequencing}

Genomic DNA was extracted from whole blood. For the 1193 patients and controls included, the whole exome $(N=687)$ or whole genome $(N=506)$ was sequenced. We used the Genome Analysis Software Kit (GATK) (version 3.4-46 or 4) best-practice pipeline to analyze our WES data (30). We aligned the reads obtained with the human reference genome (hg19), using the maximum exact matches algorithm in the Burrows-Wheeler Aligner (BWA) (31). PCR duplicates were removed with Picard tools (picard.sourceforge.net). The GATK base quality score recalibrator was applied to correct sequencing artifacts.

All the variants were manually curated using IGV and confirmed to affect the main functional protein isoform by checking the protein sequence before inclusion in further analyzes. The main functional protein isoforms are: TLR3 (NM_003265), UNC93B1 (NM_030930.4), TICAM1 (NM_182919), TRAF3 (NM_145725.2), TBK1 (NM_013254.4), IRF3 (NM_001571), IRF7 (NM_001572.5), IFNAR1 (NM_000629.3), IFNAR2 (NM_001289125.3), STAT1 (NM_007315.4), STAT2 (NM_005419.4), IRF9 (NM_006084.5). The analysis of IKBKG was customized to unmask the duplicated region in IKBKG using a specific pipeline previously described (32). We searched the NGS data for deletions in the 13 genes of interest, using both the HMZDelFinder (33) and CANOES (34) algorithms.

\section{Statistical analysis}

We performed an enrichment analysis on our cohort of 659 patients with life-threatening COVID-19 pneumonia and 534 SARSCoV2 infected controls, focusing on 12 autosomal IFN-related genes. We considered variants that were pLOF, with a MAF lower than 0.001 (gnomAD v2.1.1) after experimentally demonstrating that all the pLOF variants seen in the cases were actually LOF. We compared the proportion of individuals carrying at least one pLOF variant of the 12 autosomal genes in cases and controls by means of logistic regression with the likelihood ratio test. We accounted for the ethnic heterogeneity of the cohorts by including the first three principal components of the PCA in the logistic regression model. PC adjustment is a common and efficient strategy for accounting for different ancestries of patients and controls in the study of rare variants (35-38). We checked that our adjusted burden test was well calibrated, by also performing an analysis of enrichment in rare (MAF < 0.001) synonymous variants of the 12 genes. PCA was performed with Plink v1.9 software on Whole-Exome and Whole-Genome Sequencing data and 1000 Genomes (1kG) Project phase 3 public database as reference, using 27,480 exonic variants with a minor allele frequency $>0.01$ and a call rate $>0.99$. The odds ratio was also estimated by logistic regression and adjusted for ethnic heterogeneity.

\section{Reporter assays}

Cell lines or SV40-Fib cells with known defects were transiently or stably transfected with wild-type, mutant variants, IFN- $\beta$ - or ISRE-firefly luciferase reporter, and pRL-TK-Renilla luciferase reporter. Reporter activity was measured with the Dual-Luciferase Reporter Assay System (Promega Corporation), according to the manufacturer's instructions. Firefly luciferase activity was normalized against Renilla luciferase activity and expressed as a fold-change were calculated. TRAF3-deficient HEK293T cells were kindly provided by Dr. Maria Romanelli (39).

\section{pDC activation by SARS-CoV-2 and cytokine production}

pDCs from an IRF7 ${ }^{-1-}$ patient and a healthy donor matched for age and sex were cultured in the presence of medium alone, influenza virus (Charles River, A/PR/8/34, $2 \mu \mathrm{g} / \mathrm{mL}$ ), or the SARSCoV-2 primary strain 220_95 (GISAID accession ID: EPI_ISL_469284) at a multiplicity of infection (MOI) of 2. After 12 hours of culture, pDC supernatant was collected for cytokine quantification. IFN- $\alpha 2$ levels were measured in BD cytometric bead arrays (CBAs), in accordance with the manufacturer's protocol, with a $20 \mathrm{pg} / \mathrm{mL}$ detection limit. IFN- $\lambda 1$ secretion was measured in an enzyme-linked immunosorbent assay (ELISA) (R\&D Systems, DuoSet DY7246), in accordance with the manufacturer's instructions.

\section{SARS-CoV-2 infection in patient SV4O-Fib}

To make patients-derived fibroblasts permissive to SARS-CoV-2 infection, we delivered human ACE2 and TMPRSS2 cDNA to cells by lentivirus transduction using a modified SCRPSY vector (GenBank: KT368137.1). SARS-CoV-2, strain USA-WA1/2020, was obtained from BEI Resources. ACE2/TMPRSS2-transduced cells were either left untreated or treated with $500 \mathrm{U} / \mathrm{ml}$ IFN- $\beta$ (PBL Assay Science, cat. \#11415-1) four hours prior to infection. Cells were infected with SARS-CoV-2 (MOI = 0.5) for one hour at $37^{\circ} \mathrm{C}$. After 24 hours of infection, cells were fixed and taken out of the BSL3 for staining.

After fixation, cells were stained with SARS-CoV-2 and ACE2 primary antibodies $(0.5 \mu \mathrm{g} / \mathrm{ml}$ and $1 \mu \mathrm{g} / \mathrm{ml}$, respectively). Primary antibodies: SARS-CoV-2, human monoclonal anti-SpikeSARS-CoV-2 C121 (40); ACE2, mouse monoclonal Alexa Fluor 488-conjugated Antibody (R\&D systems, cat. \# FAB9332G100UG). Images were acquired with an ImageXpress Micro XLS microscope (Molecular Devices) using the $4 \mathrm{X}$ objective. The MetaXpress software (Molecular Devices) produced single cell mean fluorescence intensity (MFI) values.

Data analysis on single cell MFI values was done in the $\mathrm{R}$ environment (v4.0.2). ACE2/TMPRSS2-transduced cells were classified ACE2 positive when the ACE2 log MFI was superior to the log mean MFI of mock-transduced cells plus 2.5 standard deviations. We excluded all wells with less than 150 ACE2positive cells before SARS-CoV-2 scoring. ACE2-expressing cells were classified SARS-CoV-2 positive when the fluorescence intensity value was superior to the mean fluorescence intensity of mock-infected cells plus 4 standard deviations. The median SARS-CoV-2 MFI and percentage SARS-CoV-2 positive cells were calculated for each well (independent infection).

\section{Single-molecule array (Simoa) IFN- $\alpha$ digital ELISA}

Serum IFN- $\alpha$ concentrations were determined with Simoa technology, with reagents and procedures obtained from Quanterix Corporation (Quanterix SimoaTM IFNa Reagent Kit, Lexington, MA, USA). According to the manufacturer's instructions, the working dilutions were 1:2 for all sera, in working volumes of 170 $\mu \mathrm{L}$. 


\section{REFERENCES AND NOTES}

1. D. M. Morens, A. S. Fauci, Emerging pandemic diseases: How we got to COVID-19. Cell 182, 1077-1092 (2020). doi:10.1016/i.cell.2020.08.021 Medline

2. J. L. Casanova, L. Abel, Lethal Infectious Diseases as Inborn Errors of Immunity: Toward a Synthesis of the Germ and Genetic Theories. Annu. Rev. Pathol. (2020). Medline

3. J. L. Casanova, L. Abel, The human genetic determinism of life-threatening infectious diseases: Genetic heterogeneity and physiological homogeneity? Hum. Genet. 139, 681-694 (2020). doi:10.1007/s00439-020-02184-w Medline

4. J. L. Casanova, H. C. Su; COVID Human Genetic Effort, A global effort to define the human genetics of protective immunity to SARS-CoV-2 infection. Cell 181, 11941199 (2020). doi:10.1016/i.cell.2020.05.016 Medline

5. Q. Zhang, Human genetics of life-threatening influenza pneumonitis. Hum. Genet. 139, 941-948 (2020). doi:10.1007/s00439-019-02108-3 Medline

6. H. K. Lim, S. X. L. Huang, J. Chen, G. Kerner, O. Gilliaux, P. Bastard, K. Dobbs, N. Hernandez, N. Goudin, M. L. Hasek, E. J. García Reino, F. G. Lafaille, L. Lorenzo, P. Luthra, T. Kochetkov, B. Bigio, S. Boucherit, F. Rozenberg, C. Vedrinne, M. D. Keller, Y. Itan, A. García-Sastre, M. Celard, J. S. Orange, M. J. Ciancanelli, I. Meyts, Q. Zhang, L. Abel, L. D. Notarangelo, H.-W. Snoeck, J.-L. Casanova, S.-Y. Zhang, Severe influenza pneumonitis in children with inherited TLR3 deficiency. J. Exp. Med. 216, 2038-2056 (2019). doi:10.1084/jem.20181621 Medline

7. M. J. Ciancanelli, S. X. L. Huang, P. Luthra, H. Garner, Y. Itan, S. Volpi, F. G. Lafaille, C. Trouillet, M. Schmolke, R. A. Albrecht, E. Israelsson, H. K. Lim, M. Casadio, T. Hermesh, L. Lorenzo, L. W. Leung, V. Pedergnana, B. Boisson, S. Okada, C. Picard, B. Ringuier, F. Troussier, D. Chaussabel, L. Abel, I. Pellier, L. D. Notarangelo, A. García-Sastre, C. F. Basler, F. Geissmann, S.-Y. Zhang, H.-W. Snoeck, J.-L. Casanova, Life-threatening influenza and impaired interferon amplification in human IRF7 deficiency. Science 348, 448-453 (2015). doi:10.1126/science.aaa1578 Medline

8. N. Hernandez, I. Melki, H. Jing, T. Habib, S. S. Y. Huang, J. Danielson, T. Kula, S. Drutman, S. Belkaya, V. Rattina, L. Lorenzo-Diaz, A. Boulai, Y. Rose, N. Kitabayashi, M. P. Rodero, C. Dumaine, S. Blanche, M.-N. Lebras, M. C. Leung, L. S. Mathew, B. Boisson, S.-Y. Zhang, S. Boisson-Dupuis, S. Giliani, D. Chaussabel, L. D. Notarangelo, S. J. Elledge, M. J. Ciancanelli, L. Abel, Q. Zhang, N. Marr, Y. J. Crow, H. C. Su, J.-L. Casanova, Life-threatening influenza pneumonitis in a child with inherited IRF9 deficiency. J. Exp. Med. 215, 2567-2585 (2018). doi:10.1084/jem.20180628 Medline

9. V. Sancho-Shimizu, R. Pérez de Diego, L. Lorenzo, R. Halwani, A. Alangari, E. Israelsson, S. Fabrega, A. Cardon, J. Maluenda, M. Tatematsu, F. Mahvelati, M. Herman, M. Ciancanelli, Y. Guo, Z. AISum, N. Alkhamis, A. S. Al-Makadma, A. Ghadiri, S. Boucherit, S. Plancoulaine, C. Picard, F. Rozenberg, M. Tardieu, P. Lebon, E. Jouanguy, N. Rezaei, T. Seya, M. Matsumoto, D. Chaussabel, A. Puel, S.Y. Zhang, L. Abel, S. Al-Muhsen, J.-L. Casanova, Herpes simplex encephalitis in children with autosomal recessive and dominant TRIF deficiency. J. Clin. Invest. 121, 4889-4902 (2011). doi:10.1172/JCl59259 Medline

10. A. Casrouge, S.-Y. Zhang, C. Eidenschenk, E. Jouanguy, A. Puel, K. Yang, A. Alcais C. Picard, N. Mahfoufi, N. Nicolas, L. Lorenzo, S. Plancoulaine, B. Sénéchal, F. Geissmann, K. Tabeta, K. Hoebe, X. Du, R. L. Miller, B. Héron, C. Mignot, T. B. de Villemeur, P. Lebon, O. Dulac, F. Rozenberg, B. Beutler, M. Tardieu, L. Abel, J.-L. Casanova, Herpes simplex virus encephalitis in human UNC-93B deficiency. Science 314, 308-312 (2006). doi:10.1126/science.1128346 Medline

11. R. Pérez de Diego, V. Sancho-Shimizu, L. Lorenzo, A. Puel, S. Plancoulaine, C Picard, M. Herman, A. Cardon, A. Durandy, J. Bustamante, S. Vallabhapurapu, J. Bravo, K. Warnatz, Y. Chaix, F. Cascarrigny, P. Lebon, F. Rozenberg, M. Karin, M. Tardieu, S. Al-Muhsen, E. Jouanguy, S.-Y. Zhang, L. Abel, J.-L. Casanova, Human TRAF3 adaptor molecule deficiency leads to impaired Toll-like receptor 3 response and susceptibility to herpes simplex encephalitis. Immunity 33, 400411 (2010). doi:10.1016/j.immuni.2010.08.014 Medline

12. M. Herman, M. Ciancanelli, Y.-H. Ou, L. Lorenzo, M. Klaudel-Dreszler, E. Pauwels, V. Sancho-Shimizu, R. Pérez de Diego, A. Abhyankar, E. Israelsson, Y. Guo, A. Cardon, F. Rozenberg, P. Lebon, M. Tardieu, E. Heropolitańska-Pliszka, D. Chaussabel, M. A. White, L. Abel, S.-Y. Zhang, J.-L. Casanova, Heterozygous TBK1 mutations impair TLR3 immunity and underlie herpes simplex encephalitis of childhood. J. Exp. Med. 209, 1567-1582 (2012). doi:10.1084/jem.20111316 Medline

13. L. L. Andersen, N. Mørk, L. S. Reinert, E. Kofod-Olsen, R. Narita, S. E. Jørgensen, K. A. Skipper, K. Höning, H. H. Gad, L. Østergaard, T. F. Ørntoft, V. Hornung, S. R. Paludan, J. G. Mikkelsen, T. Fujita, M. Christiansen, R. Hartmann, T. H. Mogensen,
Functional IRF3 deficiency in a patient with herpes simplex encephalitis. J. Exp. Med. 212, 1371-1379 (2015). doi:10.1084/jem.20142274 Medline

14. M. Audry, M. Ciancanelli, K. Yang, A. Cobat, H.-H. Chang, V. Sancho-Shimizu, L. Lorenzo, T. Niehues, J. Reichenbach, X.-X. Li, A. Israel, L. Abel, J.-L. Casanova, S.$\mathrm{Y}$. Zhang, E. Jouanguy, A. Puel, NEMO is a key component of NF-kB- and IRF-3dependent TLR3-mediated immunity to herpes simplex virus. J. Allergy Clin. Immunol. 128, 610-617.e4 (2011). doi:10.1016/j.jaci.2011.04.059 Medline

15. N. Hernandez, G. Bucciol, L. Moens, J. Le Pen, M. Shahrooei, E. Goudouris, A. Shirkani, M. Changi-Ashtiani, H. Rokni-Zadeh, E. H. Sayar, I. Reisli, A. Lefevre-Utile, D. Zijlmans, A. Jurado, R. Pholien, S. Drutman, S. Belkaya, A. Cobat, R. Boudewijns, D. Jochmans, J. Neyts, Y. Seeleuthner, L. Lorenzo-Diaz, C. Enemchukwu, I. Tietjen, H.-H. Hoffmann, M. Momenilandi, L. Pöyhönen, M. M. Siqueira, S. M. B. de Lima, D. C. de Souza Matos, A. Homma, M. L. S. Maia, T. A. da Costa Barros, P. M. N. de Oliveira, E. C. Mesquita, R. Gijsbers, S.-Y. Zhang, S. J. Seligman, L. Abel, P. Hertzog, N. Marr, R. M. Martins, I. Meyts, Q. Zhang, M. R. MacDonald, C. M. Rice, J.-L. Casanova, E. Jouanguy, X. Bossuyt, Inherited IFNAR1 deficiency in otherwise healthy patients with adverse reaction to measles and yellow fever live vaccines. J. Exp. Med. 216, 2057-2070 (2019). doi:10.1084/iem.20182295 Medline

16. C. J. Duncan, S. M. B. Mohamad, D. F. Young, A. J. Skelton, T. R. Leahy, D. C. Munday, K. M. Butler, S. Morfopoulou, J. R. Brown, M. Hubank, J. Connell, P. J. Gavin, C. McMahon, E. Dempsey, N. E. Lynch, T. S. Jacques, M. Valappil, A. J. Cant, J. Breuer, K. R. Engelhardt, R. E. Randall, S. Hambleton, Human IFNAR2 deficiency: Lessons for antiviral immunity. Sci. Transl. Med. 7, 307ra154 (2015) doi:10.1126/scitranslmed.aac4227 Medline

17. S. Dupuis, E. Jouanguy, S. Al-Hajiar, C. Fieschi, I. Z. Al-Mohsen, S. Al-Jumaah, K. Yang, A. Chapgier, C. Eidenschenk, P. Eid, A. Al Ghonaium, H. Tufenkeji, H. Frayha, S. Al-Gazlan, H. Al-Rayes, R. D. Schreiber, I. Gresser, J.-L. Casanova, Impaired response to interferon-alpha/beta and lethal viral disease in human STAT1 deficiency. Nat. Genet. 33, 388-391 (2003). doi:10.1038/ng1097 Medline

18. S. Hambleton, S. Goodbourn, D. F. Young, P. Dickinson, S. M. B. Mohamad, M. Valappil, N. McGovern, A. J. Cant, S. J. Hackett, P. Ghazal, N. V. Morgan, R. E. Randall, STAT2 deficiency and susceptibility to viral illness in humans. Proc. Natl. Acad. Sci. U.S.A. 110, 3053-3058 (2013). doi:10.1073/pnas.1220098110 Medline

19. R. Döffinger, A. Smahi, C. Bessia, F. Geissmann, J. Feinberg, A. Durandy, C. Bodemer, S. Kenwrick, S. Dupuis-Girod, S. Blanche, P. Wood, S. H. Rabia, D. J. Headon, P. A. Overbeek, F. Le Deist, S. M. Holland, K. Belani, D. S. Kumararatne, A. Fischer, R. Shapiro, M. E. Conley, E. Reimund, H. Kalhoff, M. Abinun, A. Munnich, A. Israël, G. Courtois, J.-L. Casanova, X-linked anhidrotic ectodermal dysplasia with immunodeficiency is caused by impaired NF-kappaB signaling. Nat. Genet. 27, 277-285 (2001). doi:10.1038/85837 Medline

20. S. Y. Zhang, E. Jouanguy, S. Ugolini, A. Smahi, G. Elain, P. Romero, D. Segal, V. Sancho-Shimizu, L. Lorenzo, A. Puel, C. Picard, A. Chapgier, S. Plancoulaine, M. Titeux, C. Cognet, H. von Bernuth, C.-L. Ku, A. Casrouge, X.-X. Zhang, L. Barreiro, J. Leonard, C. Hamilton, P. Lebon, B. Héron, L. Vallée, L. Quintana-Murci, A. Hovnanian, F. Rozenberg, E. Vivier, F. Geissmann, M. Tardieu, L. Abel, J.-L. Casanova, TLR3 deficiency in patients with herpes simplex encephalitis. Science 317, 1522-1527 (2007). doi:10.1126/science.1139522 Medline

21. G. Zhang, N. A. deWeerd, S. A. Stifter, L. Liu, B. Zhou, W. Wang, Y. Zhou, B. Ying, X. Hu, A. Y. Matthews, M. Ellis, J. A. Triccas, P. J. Hertzog, W. J. Britton, X. Chen, C G. Feng, A proline deletion in IFNAR1 impairs IFN-signaling and underlies increased resistance to tuberculosis in humans. Nat. Commun. 9, 85 (2018). doi:10.1038/s41467-017-02611-z Medline

22. M. M. Thomsen, S. E. Jørgensen, M. Storgaard, L. S. Kristensen, J. Gjedsted, M. Christiansen, H. H. Gad, R. Hartmann, T. H. Mogensen, Identification of an IRF3 variant and defective antiviral interferon responses in a patient with severe influenza. Eur. J. Immunol. 49, 2111-2114 (2019). doi:10.1002/eji.201848083 Medline

23. M. M. Thomsen, S. E. Jørgensen, H. H. Gad, M. Storgaard, J. Gjedsted, M. Christiansen, R. Hartmann, T. H. Mogensen, Defective interferon priming and impaired antiviral responses in a patient with an IRF7 variant and severe influenza. Med. Microbiol. Immunol. (Berl.) 208, 869-876 (2019). doi:10.1007/s00430-01900623-8 Medline

24. S. G. Tangye, W. Al-Herz, A. Bousfiha, T. Chatila, C. Cunningham-Rundles, A. Etzioni, J. L. Franco, S. M. Holland, C. Klein, T. Morio, H. D. Ochs, E. Oksenhendler, C. Picard, J. Puck, T. R. Torgerson, J.-L. Casanova, K. E. Sullivan, Human inborn errors of immunity: 2019 update on the classification from the International Union of Immunological Societies Expert Committee. J. Clin. Immunol. 40, 24-64 (2020). doi:10.1007/s10875-019-00737-x Medline 
25. A. Bousfiha, L. Jeddane, C. Picard, W. Al-Herz, F. Ailal, T. Chatila, C. CunninghamRundles, A. Etzioni, J. L. Franco, S. M. Holland, C. Klein, T. Morio, H. D. Ochs, E. Oksenhendler, J. Puck, T. R. Torgerson, J.-L. Casanova, K. E. Sullivan, S. G. Tangye, Human Inborn Errors of Immunity: 2019 Update of the IUIS Phenotypical Classification. J. Clin. Immunol. 40, 66-81 (2020). doi:10.1007/s10875-02000758-x Medline

26. L. D. Notarangelo, R. Bacchetta, J.-L. Casanova, H. C. Su, Human inborn errors of immunity: An expanding universe. Sci. Immunol. 5, eabb1662 (2020). do::10.1126/sciimmunol.abb1662 Medline

27. J. Hadjadj, N. Yatim, L. Barnabei, A. Corneau, J. Boussier, N. Smith, H. Péré, B. Charbit, V. Bondet, C. Chenevier-Gobeaux, P. Breillat, N. Carlier, R. Gauzit, C. Morbieu, F. Pène, N. Marin, N. Roche, T.-A. Szwebel, S. H. Merkling, J.-M. Treluyer, D. Veyer, L. Mouthon, C. Blanc, P.-L. Tharaux, F. Rozenberg, A. Fischer, D. Duffy, F. Rieux-Laucat, S. Kernéis, B. Terrier, Impaired type I interferon activity and inflammatory responses in severe COVID-19 patients. Science 369, 718-724 (2020). doi:10.1126/science abc6027 Medline

28. S. Trouillet-Assant, S. Viel, A. Gaymard, S. Pons, J.-C. Richard, M. Perret, M. Villard, K. Brengel-Pesce, B. Lina, M. Mezidi, L. Bitker, A. Belot; COVID HCL Study Group, Type I IFN immunoprofiling in COVID-19 patients. J. Allergy Clin. Immunol. 146, 206-208.e2 (2020). doi:10.1016/i.jaci.2020.04.029 Medline

29. P. Bastard, L. B. Rosen, Q. Zhang, E. Michailidis, H.-H. Hoffmann, Y. Zhang, K. Dorgham, Q. Philippot, J. Rosain, V. Béziat, J. Manry, E. Shaw, L. Haljasmägi, P. Peterson, L. Lorenzo, L. Bizien, S. Trouillet-Assant, K. Dobbs, A. Almeida de Jesus, A. Belot, A. Kallaste, E. Catherinot, Y. Tandjaoui-Lambiotte, J. Le Pen, G. Kerner, B. Bigio, Y. Seeleuthner, R. Yang, A. Bolze, A. N. Spaan, O. M. Delmonte, M. S. Abers, A. Aiuti, G. Casari, V. Lampasona, L. Piemonti, F. Ciceri, K. Bilguvar, R. P. Lifton, M. Vasse, D. M. Smadja, M. Migaud, J. Hadjadj, B. Terrier, D. Duffy, L. Quintana-Murci, D. van de Beek, L. Roussel, D. C. Vinh, S. G. Tangye, F. Haerynck, D. Dalmau, J. Martinez-Picado, P. Brodin, M. C. Nussenzweig, S. Boisson-Dupuis, C. Rodríguez-Gallego, G. Vogt, T. H. Mogensen, A. J. Oler, J. Gu, P. D. Burbelo, J. Cohen, A. Biondi, L. R. Bettini, M. D'Angio, P. Bonfanti, P. Rossignol, J. Mayaux, F. Rieux-Laucat, E. S. Husebye, F. Fusco, M. V. Ursini, L. Imberti, A. Sottini, S. Paghera, E. Quiros-Roldan, C. Rossi, R. Castagnoli, D. Montagna, A. Licari, G. L. Marseglia, X. Duval, J. Ghosn, HGID Lab, NIAID-USUHS Immune Response to COVID Group, COVID Clinicians, COVID-STORM Clinicians, Imagine COVID Group, French COVID Cohort Study Group, The Milieu Intérieur Consortium, CoV-Contact Cohort, Amsterdam UMC Covid-19 Biobank, COVID Human Genetic Effort, J. S. Tsang, R. Goldbach-Mansky, K. Kisand, M. S. Lionakis, A. Puel, S.-Y. Zhang, S. M. Holland, G. Gorochov, E. Jouanguy, C. M. Rice, A. Cobat, L. D. Notarangelo, L. Abel, H. C. Su, J.-L. Casanova, Auto-antibodies against type I IFNs in patients with lifethreatening COVID-19. Science 10.1126/science.abd4585 (2020).

30. M. A. DePristo, E. Banks, R. Poplin, K. V. Garimella, J. R. Maguire, C. Hartl, A. A. Philippakis, G. del Angel, M. A. Rivas, M. Hanna, A. McKenna, T. J. Fennell, A. M. Kernytsky, A. Y. Sivachenko, K. Cibulskis, S. B. Gabriel, D. Altshuler, M. J. Daly, A framework for variation discovery and genotyping using next-generation DNA sequencing data. Nat. Genet. 43, 491-498 (2011). doi:10.1038/ng.806 Medline

31. H. Li, R. Durbin, Fast and accurate short read alignment with Burrows-Wheeler transform. Bioinformatics 25, 1754-1760 (2009) doi:10.1093/bioinformatics/btp324 Medline

32. B. Boisson, Y. Honda, M. Ajiro, J. Bustamante, M. Bendavid, A. R. Gennery, Y. Kawasaki, J. Ichishima, M. Osawa, H. Nihira, T. Shiba, T. Tanaka, M. Chrabieh, B. Bigio, H. Hur, Y. Itan, Y. Liang, S. Okada, K. Izawa, R. Nishikomori, O. Ohara, T. Heike, L. Abel, A. Puel, M. K. Saito, J.-L. Casanova, M. Hagiwara, T. Yasumi, Rescue of recurrent deep intronic mutation underlying cell type-dependent quantitative NEMO deficiency. J. Clin. Invest. 129, 583-597 (2019). doi:10.1172/JCl124011 Medline

33. T. Gambin, Z. C. Akdemir, B. Yuan, S. Gu, T. Chiang, C. M. B. Carvalho, C. Shaw, S Jhangiani, P. M. Boone, M. K. Eldomery, E. Karaca, Y. Bayram, A. Stray-Pedersen, D. Muzny, W. L. Charng, V. Bahrambeigi, J. W. Belmont, E. Boerwinkle, A. L. Beaudet, R. A. Gibbs, J. R. Lupski, Homozygous and hemizygous CNV detection from exome sequencing data in a Mendelian disease cohort. Nucleic Acids Res. 45, 1633-1648 (2017). Medline

34. D. Backenroth, J. Homsy, L. R. Murillo, J. Glessner, E. Lin, M. Brueckner, R. Lifton, E. Goldmuntz, W. K. Chung, Y. Shen, CANOES: Detecting rare copy number variants from whole exome sequencing data. Nucleic Acids Res. 42, e97 (2014). doi:10.1093/nar/gku345 Medline

35. M. Bouaziz, J. Mullaert, B. Bigio, Y. Seeleuthner, J.-L. Casanova, A. Alcais, L. Abel, A. Cobat, Controlling for human population stratification in rare variant association studies. bioRxiv 969477 [Preprint]. 28 February 2020. https://doi.org/10.1101/2020.02.28.969477.

36. E. Persyn, R. Redon, L. Bellanger, C. Dina, The impact of a fine-scale population stratification on rare variant association test results. PLOS ONE 13, e0207677 (2018). doi:10.1371/journal.pone.0207677 Medline

37. Y. Zhang, X. Shen, W. Pan, Adjusting for population stratification in a fine scale with principal components and sequencing data. Genet. Epidemiol. 37, 787-801 (2013). doi:10.1002/gepi.21764 Medline

38. S. Boisson-Dupuis, N. Ramirez-Alejo, Z. Li, E. Patin, G. Rao, G. Kerner, C. K. Lim, D. N. Krementsov, N. Hernandez, C. S. Ma, Q. Zhang, J. Markle, R. MartinezBarricarte, K. Payne, R. Fisch, C. Deswarte, J. Halpern, M. Bouaziz, J. Mulwa, D. Sivanesan, T. Lazarov, R. Naves, P. Garcia, Y. Itan, B. Boisson, A. Checchi, F. JabotHanin, A. Cobat, A. Guennoun, C. C. Jackson, S. Pekcan, Z. Caliskaner, J. Inostroza, B. T. Costa-Carvalho, J. A. T. de Albuquerque, H. Garcia-Ortiz, L. Orozco, T. Ozcelik, A. Abid, I. A. Rhorfi, H. Souhi, H. N. Amrani, A. Zegmout, F. Geissmann, S. W. Michnick, I. Muller-Fleckenstein, B. Fleckenstein, A. Puel, M. J. Ciancanelli, N. Marr, H. Abolhassani, M. E. Balcells, A. Condino-Neto, A. Strickler, K. Abarca, C. Teuscher, H. D. Ochs, I. Reisli, E. H. Sayar, J. El-Baghdadi, J. Bustamante, L. Hammarström, S. G. Tangye, S. Pellegrini, L. Quintana-Murci, L. Abel, J.-L. Casanova, Tuberculosis and impaired IL-23-dependent IFN- $\gamma$ immunity in humans homozygous for a common TYK2 missense variant. Sci. Immunol. 3 , eaau8714 (2018). doi:10.1126/sciimmunol.aau8714 Medline

39. S. Fochi, E. Bergamo, M. Serena, S. Mutascio, C. Journo, R. Mahieux, V. Ciminale, U. Bertazzoni, D. Zipeto, M. G. Romanelli, TRAF3 Is Required for NF-кB Pathway Activation Mediated by HTLV Tax Proteins. Front. Microbiol. 10, 1302 (2019). doi:10.3389/fmicb.2019.01302 Medline

40. D. F. Robbiani, C. Gaebler, F. Muecksch, J. C. C. Lorenzi, Z. Wang, A. Cho, M. Agudelo, C. O. Barnes, A. Gazumyan, S. Finkin, T. Hägglöf, T. Y. Oliveira, C. Viant, A. Hurley, H.-H. Hoffmann, K. G. Millard, R. G. Kost, M. Cipolla, K. Gordon, F. Bianchini, S. T. Chen, V. Ramos, R. Patel, J. Dizon, I. Shimeliovich, P. Mendoza, H. Hartweger, L. Nogueira, M. Pack, J. Horowitz, F. Schmidt, Y. Weisblum, E. Michailidis, A. W. Ashbrook, E. Waltari, J. E. Pak, K. E. Huey-Tubman, N. Koranda, P. R. Hoffman, A. P. West Jr., C. M. Rice, T. Hatziioannou, P. J. Bjorkman, P. D. Bieniasz, M. Caskey, M. C. Nussenzweig, Convergent antibody responses to SARS-CoV-2 in convalescent individuals. Nature 584, 437-442 (2020). doi:10.1038/s41586-020-2456-9 Medline

41. M. Ogishi, R. Yang, C. Gruber, S. Pelham, A. N. Spaan, J. Rosain, M. Chbihi, J. E. Han, V. K. Rao, L. Kainulainen, J. Bustamante, B. Boisson, D. Bogunovic, S. Boisson-Dupuis, J.-L. Casanova, Multi-batch cytometry data integration for optimal immunophenotyping. bioRxiv 202432 [Preprint]. 15 July 2020. https://doi.org/10.1101/2020.07.14.202432.

42. P. Meade, G. Kuan, S. Strohmeier, H. E. Maier, F. Amanat, A. Balmaseda, K. Ito, E. Kirkpatrick, A. Javier, L. Gresh, R. Nachbagauer, A. Gordon, F. Krammer, Influenza virus infection induces a narrow antibody response in children but a broad recall response in adults. mBio 11, e03243-19 (2020). doi:10.1128/mBio.03243-19 Medline

\section{ACKNOWLEDGMENTS}

We thank the patients, their families, and healthy donors for placing their trust in us. We thank Y. Nemirowskaya, D. Papandrea, M. Woollet, D. Liu, C. Rivalain and C. Patissier for administrative assistance; and A. Adeleye, D. Bacikova, E. McGrath Martinez, A. R. Soltis, K. Dobbs, J. Danielson, H. Matthews, and S. Weber for technical and other assistance. We thank M. M. A. Ata and F. Al Ali for their contribution to VirScan experiments. We would like to thank Dr. Stephen Elledge (Brigham and Women's Hospital and Harvard Medical School, Boston, MA) for kindly providing the VirScan phage library used in this study. We thank A. W. Ashbrook, the BSL3 manager of the Rice lab. We thank M. Lazzaro, Director of Immigration and Academic Appointments for his assistance. From JM lab, we thank W. Chung, K. Kiryluk, S. O'Byrne, D. Pendrick. J. Williamson, C. Andrews, and M. Disco. We thank Prof. Massimo Andreoni (Tor Vergata, Italy) for his clinical contribution. We thank Antonio Novelli (Bambino Gesù Hospital, Italy) for his collaboration. This study utilized the high-performance computational resources of the NIH HPC Biowulf cluster (http://hpc.nih.gov) and the Office of Cyber Infrastructure and Computational Biology (OCICB) High Performance Computing (HPC) cluster at the National Institute of Allergy and Infectious Diseases (NIAID), Bethesda, MD. The opinions and assertions expressed herein are those of the authors and are not to be construed as reflecting the views of USUHS or the United States Department of Defense. Funding: We thank the 
generous donation from Fisher Center for Alzheimer's Research Foundation for our research. The Laboratory of Human Genetics of Infectious Diseases is supported by the Howard Hughes Medical Institute, the Rockefeller University, the St. Giles Foundation, the National Institutes of Health (NIH) (R01AI088364), the National Center for Advancing Translational Sciences (NCATS), NIH Clinical and Translational Science Award (CTSA) program (UL1 TR001866), a Fast Grant from Emergent Ventures, Mercatus Center at George Mason University, the Yale Center for Mendelian Genomics and the GSP Coordinating Center funded by the National Human Genome Research Institute (NHGRI) (UM1HG006504 and U24HG008956), the French National Research Agency (ANR) under the "Investments for the Future" program (ANR-10-IAHU-01), the Integrative Biology of Emerging Infectious Diseases Laboratory of Excellence (ANR-10-LABX-62IBEID), the French Foundation for Medical Research (FRM)

(EQU201903007798), the FRM and ANR GENCOVID project, ANRS-COV05, the Square Foundation, Grandir - Fonds de solidarité pour l'enfance, the SCOR Corporate Foundation for Science, Institut National de la Santé et de la Recherche Médicale (INSERM), the University of Paris. The French COVID Cohort study group was sponsored by Inserm and supported by the REACTing consortium and by a grant from the French Ministry of Health (PHRC 20-0424). Regione Lombardia, Italy (project "Risposta immune in pazienti con COVID-19 e co-morbidità"), and the Intramural Research Program of the NIAID, NIH. The laboratory of Genomes \& Cell Biology of Disease is supported by "Integrative Biology of Emerging Infectious Diseases" (grant no. ANR-10-LABX-62-IBEID), the "Fondation pour la Recherche Medicale" (grant FRM - EQU202003010193), the "Agence Nationale de la Recherche" (ANR FLASH COVID project IDISCOVR cofounded by the "Fondation pour la Recherche Médicale"), University of Paris ("Plan de Soutien Covid-19": RACPL20FIR01-COVID-SOUL). IM is a senior clinical investigator with the FWO Vlaanderen; IM and LM are supported by FWO G0C8517N - GOB5120N. The VS team was supported by "Agence Nationale de la Recherche" (ANR-17-CE15-0003, ANR-17-CE15-0003-01), and by Université de Paris "PLAN D'URGENCE COVID19". LK was supported by a fellowship from the French Ministry of Research. VS-S is supported by a UKRI Future Leaders Fellowship (MR/S032304/1). SZA-M is supported by the Elite Journals Program at King Saud University through grant number PEJP-16-107. JM lab is supported by Columbia University COVID biobank and grant: UL1TR001873. Work in the Laboratory of Virology and Infectious Disease was supported by NIH grants P01Al138398-S1, 2U19Al111825, and R01AI091707-10S1, a George Mason University Fast Grant, and the G. Harold and Leila Y. Mathers Charitable Foundation. JLP is supported by a European Molecular Biology Organization Long-Term Fellowship (ALTF 380-2018). Work at the Neurometabolic Diseases Laboratory received funding from the European Union's Horizon 2020 research and innovation program under grant agreement No 824110 (EasiGenomics grant COVID-19/ PID12342) to A.P., and Roche and Illumina Covid Match Funds to M.G.. C.R.G and colleagues are supported by clnstituto de Salud Carlos III (COV20_01333 and COV20_01334), Spanish Ministry of Science and Innovation, with the funding of European Regional Development Fund-European Social Fund -FEDER-FSE; (RTC-2017-6471-1; AEI/FEDER, UE), and Cabildo Insular de Tenerife (CGIEU0000219140 and "Apuestas científicas del ITER para colaborar en la lucha contra la COVID-19"). D.C.V. is supported by the Fonds de la recherche en santé du Québec clinician-scientist scholar program. Helen Su is adjunct faculty at the University of Pennsylvania. A-L.N. was supported by the Foundation Bettencourt Schueller. The Amsterdam UMC Covid-19 Biobank was funded by the Netherlands Organization for Health Research and Development (ZonMw, NWO-vici 91819627), The Corona Research Fund (Amsterdam UMC), Dr. J. C. Vaillantfonds, and Amsterdam UMC. Work on COVID-19 at the AG-S lab is partly supported by NIH supplements to grants U19Al135972, U19Al142733 and R35 HL135834, and to contract HHSN272201800048C, by a DoD supplement to grant W81XWH-20-1-0270, by DARPA project HR0011-19-20020, by CRIP (Center for Research on Influenza Pathogenesis), a NIAID funded Center of Excellence for Influenza Research and Surveillance (CEIRS, contract HHSN272201400008C), by an NIAID funded Collaborative Influenza Vaccine Innovation Center (SEM-CIVIC, contract 75N93019C00051) and by the generous support of the JPB Foundation, the Open Philanthropy Project (research grant 2020-215611(5384)) and anonymous donors. The Virscan analysis presented in fig. S11 was performed with financial support from Sidra Medicine. Author contributions: A.G., A.A., A.A.A., A.L.S., A-L.N., A.C., A.C., A.P., B.B., B.S.R., C.A., C.M., C.K., C.L., C.M.R., C.L.D., D.D., E.M., E.J., F.A., F.A-M., F.O., F.A., F.K., G.N., G.S., G.G., H-H.H., H.K.A.S., H.S., I.K.D.S., I.M., J.L.P., J.R., J.E.H., J.C., J.M., J.Y.,
K.D., K.B., L.A., L.L-D., L.K., L.M., L.B-M., L.B., L.D.N., M.M-V., M.C., M.O., M.C., M.N., M.F.T., M.S., M.F.A., N.M., N.S., P.B., P.M., Q.Z., Q.Z., Q.P., R.L., R.Y., S.A.T., S.Z.A-M., S.H., S.K., S.H., S.B-D., T.K., T.M., T.M., V.S-S., V.S., V.B., W.S., X.D., Y.S., Z.L. either performed or supervised experiments, generated and analyzed data, and contributed to the manuscript. A.S., A.C.U., A.B., A.O., A.P., B.B., D.V.D.B., F.R., G.K., J.M., P.Z., S-Y.Z., T.L-V., Y.S., Y.Z. performed computational analysis. A.S., A.N.S., A.M-N., A.B., C.R., D.M., D.C.V., E.Q-R., F.H., I.M., I.V., J.B., J-C.G., L.R.B., L.R., L.I., M.D., P.B., P.S-P., P-E.M., R.H., R.C., S.K., S.P., T.O., Y.TL., K.K., S.S., J.F., S.N.K. evaluated and recruited patients to COVID and/or control cohorts. Q.Z. P.B. A.C. E.J. L.A. S-Y.Z. and J-L.C. wrote the manuscript. All authors edited the manuscript. J-L.C. supervised the project. Competing interests: The authors declare no competing financial interest. Jean-Laurent Casanova is listed as an inventor on patent application US63/055, 155 filed by The Rockefeller University that encompasses aspects of this publication. Richard Lifton is a non-executive director of Roche and its subsidiary Genentech. Data and materials availability: Plasma, cells, and genomic DNA are available from Dr. Jean-Laurent Casanova/Dr. Donald Vinh under a material agreement with Rockfeller University/Research Institute-McGill University Health Centre. pSCRPSY_TMPRSS2-2A-NeoR_ACE2 and Huh-7.5 cells are available upon request from $C$. Rice under a material agreement with The Rockefeller University, or The Rockefeller University and Apath, LLC, respectively. Clinical data, DNA, and other patient samples are available from the Amsterdam UMC Covid-19 Biobank (Diederik van de Beek) under a material agreement with Amsterdam UMC. Material and reagents used are almost exclusively commercially available and non-proprietary. Requests for materals derived from human samples may be made available, subject to any underlying restrictions on such samples. Jean-Laurent Casanova can make material transfer agreements available through The Rockefeller University. Detailed genotype counts for all coding variants in the genes investigated in this manuscript are available at https://doi.org/10.5061/dryad.8pk0p2nkk. The WGS datasets used for the analyses including critical patients and asymptomatic controls described in this manuscript were deposited in $\mathrm{dbGaP}$ under accession number phs002245.v1.p1. All other data are available in manuscript or supplementary material. This work is licensed under a Creative Commons Attribution 4.0 International (CC BY 4.0) license, which permits unrestricted use, distribution, and reproduction in any medium, provided the original work is properly cited. To view a copy of this license, visit

https://creativecommons.org/licenses/by/4.0/. This license does not apply to figures/photos/artwork or other content included in the article that is credited to a third party; obtain authorization from the rights holder before using such material.

\section{COVID-STORM Clinicians}

Giuseppe Foti ${ }^{1}$, Giacomo Bellani ${ }^{1}$, Giuseppe Citerio ${ }^{1}$, Ernesto Contro ${ }^{1}$, Alberto Pesci ${ }^{2}$, Maria Grazia Valsecchi ${ }^{3}$, Marina Cazzaniga ${ }^{4}$

${ }^{1}$ Department of Emergency, Anesthesia and Intensive Care, School of Medicine and Surgery, University of Milano-Bicocca, San Gerardo Hospital, Monza, Italy. ${ }^{2}$ Department of Pneumology, School of Medicine and Surgery, University of Milano-Bicocca, San Gerardo Hospital, Monza, Italy. ${ }^{3}$ Center of Bioinformatics and Biostatistics, School of Medicine and Surgery, University of Milano-Bicocca, San Gerardo Hospital, Monza, Italy. ${ }^{4}$ Phase I Research Center, School of Medicine and Surgery, University of Milano-Bicocca, San Gerardo Hospital, Monza IT

\section{COVID Clinicians}

Jorge Abad1, Sergio Aguilera-Albesa², Ozge Metin Akcan³, Ilad Alavi Darazam ${ }^{4}$, Juan C. Aldave ${ }^{5}$, Miquel Alfonso Ramos ${ }^{6}$, Seyed Alireza Nadji', Gulsum Alkan ${ }^{8}$, Jerome Allardet-Servent ${ }^{9}$, Luis M. Allende ${ }^{10}$, Laia Alsina ${ }^{11}$, Marie-Alexandra Alyanakian ${ }^{12}$, Blanca Amador-Borrero ${ }^{13}$, Zahir Amoura ${ }^{14}$, Arnau Antoli ${ }^{15}$, Sevket Arslan ${ }^{16}$, Sophie Assant ${ }^{17}$, Terese Auguet ${ }^{18}$, Axelle Azot ${ }^{19}$, Fanny Bajolle ${ }^{20}$, Aurélie Baldolli21, Maite Ballester ${ }^{22}$, Hagit Baris Feldman ${ }^{23}$, Benoit Barrou ${ }^{24}$, Alexandra Beurton ${ }^{25}$, Agurtzane Bilbao ${ }^{26}$, Geraldine Blanchard-Rohner ${ }^{27}$, Ignacio Blanco ${ }^{1}$, Adeline Blandinières ${ }^{28}$, Daniel Blazquez-Gamero ${ }^{29}$, Marketa Bloomfield ${ }^{30}$, Mireia Bolivar-Prados ${ }^{31}$, Raphael Borie ${ }^{32}$, Cédric Bosteels ${ }^{33}$, Ahmed A. Bousfiha ${ }^{34}$, Claire Bouvattie ${ }^{35}$, Oksana Boyarchuk ${ }^{36}$, Maria Rita P. Bueno ${ }^{37}$, Jacinta Bustamante ${ }^{20}$, Juan José Cáceres Agra ${ }^{38}$, Semra Camli $^{39}$, Ruggero Capra ${ }^{40}$, Maria Carrabba ${ }^{41}$, Carlos Casasnovas ${ }^{42}$, Marion Caseris ${ }^{43}$, Martin Castelle ${ }^{44}$, Francesco Castelli ${ }^{45}$, Martín Castillo de Vera ${ }^{46}$, Mateus V. Castro ${ }^{37}$. Emilie Catherinot ${ }^{47}$, Martin Chalumeau ${ }^{48}$, Bruno Charbit ${ }^{49}$, Matthew P. Cheng ${ }^{50}$, Père 
Clavé $e^{31}$, Bonaventura Clotet ${ }^{51}$, Anna Codina ${ }^{52}$, Fatih Colkesen ${ }^{53}$, Fatma Çölkesen ${ }^{54}$, Roger Colobran ${ }^{55}$, Cloé Comarmond ${ }^{56}$, David Dalmau ${ }^{57}$, David Ross Darley ${ }^{58}$, Nicolas Dauby $^{59}$, Stéphane Dauger ${ }^{60}$, Loic de Pontua ${ }^{61}$, Amin Dehban ${ }^{62}$, Geoffroy Delplancq ${ }^{63}$, Alexandre Demoule ${ }^{64}$, Jean-Luc Dieh|65 , Stephanie Dobbelaere ${ }^{66}$, Sophie Durand ${ }^{67}$. Waleed Eldars ${ }^{68}$, Mohamed Elgamal ${ }^{69}$, Marwa H. Elnagdy ${ }^{70}$, Melike Emiroglu ${ }^{71}$, Emine Hafize Erdeniz ${ }^{72}$, Selma Erol Aytekin ${ }^{73}$, Romain Euvrard ${ }^{74}$, Recep Evcen $^{75}$, Giovanna Fabio ${ }^{41}$, Laurence Faivre ${ }^{76}$, Antonin Falck ${ }^{43}$, Muriel Fartoukh ${ }^{77}$, Morgane Faure ${ }^{78}$, Miguel Fernandez Arquero ${ }^{79}$, Carlos Flores ${ }^{80}$, Bruno Francois ${ }^{81}$, Victoria Fumadó ${ }^{82}$, Francesca Fusco ${ }^{83}$, Blanca Garcia Solis ${ }^{84}$, Pascale Gaussem ${ }^{85}$, Juana Gil-Herrera ${ }^{86}$, Laurent Gilardin ${ }^{87}$, Monica Girona Alarcon ${ }^{88}$, Mònica Girona-Alarcón ${ }^{88}$, Jean-Christophe Goffard ${ }^{89}$, Funda Gok ${ }^{90}$, Rafaela González-Montelongo ${ }^{91}$, Antoine Guerder $^{92}$, Yahya Gul ${ }^{93}$, Sukru Nail Guner ${ }^{93}$, Marta Gut ${ }^{94}$, Jérôme Hadjadj ${ }^{95}$, Filomeen Haerynck ${ }^{96}$, Rabih Halwani ${ }^{97}$, Lennart Hammarström ${ }^{98}$, Nevin Hatipoglu ${ }^{99}$, Elisa Hernandez-Brito ${ }^{100}$, Cathérine Heymans ${ }^{101}$, María Soledad Holanda-Peña ${ }^{102}$, Juan Pablo Horcajada ${ }^{103}$, Levi Hoste ${ }^{104}$, Eric Hoste ${ }^{105}$, Sami Hraiech ${ }^{106}$, Linda Humbert ${ }^{107}$, Alejandro D. Iglesias ${ }^{108}$, Antonio Íñigo-Campos ${ }^{91}$, Matthieu Jamme ${ }^{109}$, María Jesús Arranz ${ }^{110}$, Iolanda Jordan ${ }^{111}$, Philippe Jorens ${ }^{112}$, Fikret Kanat ${ }^{113}$, Hasan Kapak $\mid 1^{114}$, Iskender Kara ${ }^{115}$, Adem Karbuz ${ }^{116}$, Kadriye Kart Yasar ${ }^{117}$, Sevgi Keles ${ }^{118}$, Yasemin Kendir Demirko ${ }^{119}$, Adam Klocperk ${ }^{120}$, Zbigniew J. Król121, Paul Kuentz ${ }^{122}$, Yat Wah M. Kwan ${ }^{123}$, Jean-Christophe Lagier ${ }^{124}$, Bart N. Lambrecht ${ }^{33}$, Yu-Lung LAUU' ${ }^{125}$, Fleur Le Bourgeois $^{60}$, Yee-Sin Leo ${ }^{126}$, Rafael Leon Lopez ${ }^{127}$, Daniel Leung ${ }^{125}$, Michael Levin ${ }^{128}$, Michael Levy ${ }^{60}$, Romain Lévy ${ }^{20}$, Zhi Li49, Agnes Linglart ${ }^{129}$, Bart Loeys ${ }^{130}$, José M. Lorenzo-Salazar ${ }^{91}$, Céline Louapre ${ }^{131}$, Catherine Lubetzki ${ }^{131}$, Charles-Edouard Luyt $t^{132}$, David C. Lye ${ }^{133}$, Davood Mansouri ${ }^{134}$, Majid Marjani ${ }^{135}$, Jesus Marquez Pereira ${ }^{136}$, Andrea Martin ${ }^{137}$, David Martínez Pueyo $0^{138}$, Javier Martinez-Picado ${ }^{139}$, Iciar Marzana ${ }^{140}$ Alexis Mathian ${ }^{14}$, Larissa R.B. Matos ${ }^{37}$, Gail V. Matthews ${ }^{141}$, Julien Mayaux ${ }^{142}$, JeanLouis Mège ${ }^{143}$, Isabelle Melki ${ }^{144}$, Jean-François Meritet ${ }^{145}$, Ozge Metin ${ }^{146}$, Isabelle Meyts $^{147}$. Mehdi Mezidi148, Isabelle Migeotte ${ }^{149}$, Maude Millereux ${ }^{150}$, Tristan Mirault ${ }^{151}$, Clotilde Mircher ${ }^{67}$, Mehdi Mirsaeidi ${ }^{152}$, Abián Montesdeoca Melián ${ }^{153}$, Antonio Morales Martine $z^{154}$, Pierre Morange ${ }^{155}$, Demence Mordacq ${ }^{107}$, Guillaume Morelle ${ }^{156}$. Stéphane Mouly ${ }^{13}$, Adrián Muñoz-Barrera ${ }^{91}$, Leslie Naesens ${ }^{157}$, Cyril Nafati158, João Farela Neves ${ }^{159}$, Lisa F.P. Ng ${ }^{160}$, Yeray Novoa Medina ${ }^{161}$, Esmeralda Nuñez Cuadros ${ }^{162}$, J. Gonzalo Ocejo-Vinyals ${ }^{163}$, Zerrin Orbak ${ }^{164}$, Mehdi Oualha ${ }^{20}$, Tayfun Özçelik ${ }^{165}$, Qiang Pan Hammarström ${ }^{166}$, Christophe Parizot ${ }^{142}$, Tiffany Pascreau ${ }^{167}$, Estela Paz-Artal ${ }^{168}$ Sandra Pellegrini ${ }^{49}$, Rebeca Pérez de Dieg0 ${ }^{84}$, Aurélien Philippe $\mathrm{e}^{169}$, Quentin Philippot $^{77}$, Laura Planas-Serra ${ }^{170}$, Dominique Ploin ${ }^{171}$, Julien Poissy ${ }^{172}$, Géraldine Poncelet ${ }^{43}$, Marie Pouletty ${ }^{173}$, Paul Quentric ${ }^{142}$, Didier Raoult ${ }^{143}$, Anne-Sophie Rebillat ${ }^{67}$, Ismail Reislili74, Pilar Ricart ${ }^{175}$, Jean-Christophe Richard ${ }^{176}$, Nadia rivet ${ }^{28}$, Jacques G. Rivière ${ }^{177}$, Gemma Rocamora Blanch ${ }^{15}$, Carlos Rodrigo ${ }^{1}$, Carlos Rodriguez-Gallego ${ }^{178}$, Agustí Rodríguez-Palmero ${ }^{179}$, Carolina Soledad Romero ${ }^{180}$, Anya Rothenbuhler ${ }^{181}$, Flore Rozenberg ${ }^{182}$, Maria Yolanda Ruiz del Prad0 ${ }^{183}$, Joan Sabater Riera ${ }^{15}$, Oliver Sanchez ${ }^{184}$, Silvia Sánchez-Ramón ${ }^{185}$, Agatha Schluter ${ }^{170}$, Matthieu Schmidt ${ }^{186}$, Cyril E. Schweitzer ${ }^{187}$, Francesco Scolari ${ }^{188}$, Anna Sediva ${ }^{189}$, Luis M. Seijo ${ }^{190}$, Damien Sene $^{13}$, Sevtap Senoglu ${ }^{117}$, Mikko Seppänen ${ }^{191}$, Alex Serra llovich ${ }^{192}$, Mohammad Shahrooei ${ }^{62}$, Hans Slabbynck ${ }^{193}$, David Smadja ${ }^{194}$, Ali Sobh ${ }^{195}$, Xavier Solanich Moreno $^{15}$, Jordi Solé-Violán ${ }^{196}$, Catherine Soler ${ }^{197}$, Pere Soler-Palacín ${ }^{137}$, Yuri Stepanovskiy ${ }^{198}$, Annabelle Stoclin ${ }^{199}$, Fabio Taccone ${ }^{149}$, Yacine Tandjaoui-Lambiotte ${ }^{200}$ Jean-Luc Taupin ${ }^{201}$, Simon J. Tavernier ${ }^{202}$, Benjamin Terrier ${ }^{203}$, Caroline Thumerelle ${ }^{107}$, Gabriele Tomasoni ${ }^{204}$, Julie Toubiana ${ }^{48}$, Josep Trenado Alvarez ${ }^{205}$, Sophie Trouillet-Assant ${ }^{206}$, Jesús Troya ${ }^{207}$. Alessandra Tucci ${ }^{208}$, Matilde Valeria Ursini ${ }^{83}$, Yurdagul Uzunhan ${ }^{209}$, Pierre Vabres ${ }^{210}$, Juan Valencia-Ramos ${ }^{211}$, Eva Van Braecke ${ }^{33}$ Stijn Van de velde ${ }^{212}$, Ana Maria Van Den Rym ${ }^{84}$, Jens Van Praet ${ }^{213}$, Isabelle Vandernoot $^{214}$, Hulya Vatansev ${ }^{215}$, Valentina Vélez-Santamaria ${ }^{42}$, Sébastien Vie ${ }^{171}$, Cédric Vi${ }^{l a i n}{ }^{216}$, Marie E. Vilaire ${ }^{67}$, Audrey Vincent ${ }^{35}$, Guillaume Voiriot ${ }^{217}$, Fanny Vuotto ${ }^{107}$, Alper Yosunkaya ${ }^{90}$, Barnaby E Young ${ }^{126}$, Fatih Yuce ${ }^{218}$, Faiez Zannad ${ }^{219}$, Mayana Zatz $^{37}$, Alexandre Belot ${ }^{220 *}$

'University Hospital and Research Institute "Germans Trias i Pujol", Badalona, Spain. ${ }^{2}$ Navarra Health Service Hospital, Pamplona, Spain. ${ }^{3}$ Division of Pediatric Infectious Diseases, Necmettin Erbakan University, Meram Medical Faculty, Konya, Turkey. ${ }^{4}$ Department of Infectious Diseases, Loghman Hakim Hospital, Shahid Beheshti University of Medical Sciences, Tehran, Iran. ${ }^{5} \mathrm{H}$ ospital Nacional Edgardo Rebagliati Martins, Lima, Peru. ${ }^{6}$ Parc Sanitari Sant Joan de Déu, Sant Boi de Llobregat Spain. ${ }^{7}$ Virology Research Center, National institutes of Tuberculosis and Lung diseases, Shahid Beheshti University of Medical Sciences, Tehran, Iran. ${ }^{8}$ Division of Pediatric Infectious Diseases, Faculty of Medicine, Selcuk University, Konya, Turkey. ${ }^{9}$ Intensive care unit, Hôpital Européen, Marseille, France. ${ }^{10}$ Immunology Department, University Hospital 12 de Octubre. Research Institute imas12. Complutense University, Madrid, Spain. ${ }^{11}$ Hospital Sant Joan de Déu, Barcelona, Spain. ${ }^{12}$ Department of
Biological Immunology, Necker Hospital for Sick Children, APHP and INEM. Paris, France. ${ }^{13}$ Internal medicine department, Hôpital Lariboisière, APHP; Université de Paris, Paris, France. ${ }^{14}$ Internal medicine department, Pitié-Salpétrière Hospital, Paris, France. ${ }^{15} \mathrm{Hospital}$ Universitari de Bellvitge, Barcelona, Spain. ${ }^{16}$ Division of Clinical Immunology and Allergy, Necmettin Erbakan University, Meram Medical Faculty, Konya, Turkey. ${ }^{17}$ Joint Research Unit, Hospices Civils de Lyon-bio Mérieux, Hospices Civils de Lyon, Lyon Sud Hospital, Lyon, France. ${ }^{18} \mathrm{Hospital} U$. de Tarragona Joan XXIII. Universitat Rovira i Virgili (URV). IISPV, Tarragona, Spain. ${ }^{19}$ Private practice, Paris, France. ${ }^{20}$ Necker Hospital for Sick Children, AP-HP, Paris, France. ${ }^{21}$ Department of Infectious Diseases, CHU de Caen, Caen, France. ${ }^{22}$ Consorcio Hospital General Universitario, Valencia, Spain. ${ }^{23}$ The Genetics Institute, Tel Aviv Sourasky Medical Center and Sackler Faculty of Medicine, Tel Aviv University, Tel Aviv, Israel. ${ }^{24}$ Dept Urology, Nephrology, Transplantation, APHP-SU, Sorbonne Université, INSERM U 1082, Paris, France. ${ }^{25}$ Service de Médecine Intensive-Réanimation et Pneumologie, APHP Hôpital Pitié-Salpêtrière, Paris, France. ${ }^{26}$ Cruces University Hospital, Bizkaia, Spain. ${ }^{27}$ Paediatric Immunology and Vaccinology Unit, Geneva University Hospitals and Faculty of Medicine, Geneva, Switzerland. ${ }^{28} \mathrm{Hematology}$, Georges Pompidou Hospital, APHP, Paris, France. ${ }^{29}$ Pediatric Infectious Diseases Unit. Instituto de Investigación 12 de Octubre (imas12). Hospital Universitario 12 de Octubre, Madrid, Spain. ${ }^{30}$ Department of Immunology, Motol University Hospital, 2nd Faculty of Medicine, Charles University, Department of Pediatrics, Thomayer's Hospital, 1st Faculty of Medicine, Charles University, Prague, Czech Republic. ${ }^{31} \mathrm{Cen}$ tro de Investigación Biomédica en Red de Enfermedades Hepàticas y Digestivas (Ciberehd). Hospital de Mataró, Consorci Sanitari del Maresme, Mataró, Spain.

${ }^{32}$ Service de Pneumologie, Hopital Bichat, APHP, Paris, France. ${ }^{33}$ Department of Pulmonology, Ghent University Hospital, Ghent, Belgium. ${ }^{34} \mathrm{Clinical}$ immunology unit, pediatric infectious disease departement, Faculty of Medicine and Pharmacy, Averroes University Hospital. LICIA Laboratoire d'immunologie clinique, d'inflammation et d'allergie, Hassann li University, Casablanca, Morocco. ${ }^{35}$ Endocrinology unit, APHP Hôpitaux Universitaires Paris-Sud, Le Kremlin-Bicêtre, France. ${ }^{36}$ Department of Children's Diseases and Pediatric Surgery. I.Horbachevsky Ternopil National Medical University, Ternopil, Ukraine. ${ }^{37}$ Human Genome and stem-cell research centerUniversity of São Paulo, São Paulo, Brazil. ${ }^{38} \mathrm{Hospital}$ Insular, Las Palmas de Gran Canaria, Spain. ${ }^{39}$ Division of Critical Care Medicine, Department of Anesthesiology and Reanimation, Konya State Hospital, Konya, Turkey. ${ }^{40}$ MS Center, Spedali Civili, Brescia, Italy. ${ }^{41}$ Fondazione IRCCS Ca' Granda Ospedale Maggiore Policlinico, Milan, Italy. ${ }^{42}$ Bellvitge University Hospital, L'Hospitalet de Llobregat, Barcelona, Spain. ${ }^{43}$ Hopital Robert Debré, Paris, France. ${ }^{44}$ Pediatric Immuno-hematology Unit, Necker Enfants Malades Hospital, AP-HP, Paris, France. ${ }^{45}$ Department of Infectious and Tropical Diseases, University of Brescia, ASST Spedali Civili di Brescia, Brescia, Italy. ${ }^{46}$ Doctoral Health Care Center, Canarian Health System, Las Palmas de Gran Canaria, Spain. ${ }^{47}$ Hôpital Foch, Suresnes, France. ${ }^{48}$ Necker Hospital for Sick Children, Paris University, AP-HP, Paris, France. ${ }^{49}$ Pasteur Institute, Paris, France. ${ }^{50}$ McGill University Health Centre, Montreal, Canada. ${ }^{51}$ University Hospital and Research Institute "Germans Trias i Pujol", IrsiCaixa AIDS Research Institute, UVic-UCC, Badalona, Spain. ${ }^{52} \mathrm{Clinical}$ Biochemistry, Pathology. Paediatric Neurology and Molecular Medicine Departments and Biobank, Institut de Recerca Sant Joan de Déu and CIBERERISCIII, Esplugues, Spain. ${ }^{53}$ Division of Clinical Immunology and Allergy, Department of Internal Medicine, Necmettin Erbakan University, Meram Medical Faculty, Konya, Turkey. ${ }^{54}$ Department of Infectious Diseases and Clinical Microbiology, Konya Training and Research Hospital, Konya, Turkey. ${ }^{55}$ Hospital Universitari Vall d'Hebron, Barcelona, Spain. ${ }^{56}$ Pitié-Salpêtrière Hospital, Paris, France. ${ }^{57}$ Fundació Docència i Recerca Mútua Terrassa, Barcelona, Spain. ${ }^{58}$ UNSW Medicine, St Vincent's Clinical School; Department of Thoracic Medicine, St Vincent's Hospital Darlinghurst, Sidney, Australia. ${ }^{59} \mathrm{CHU}$ Saint-Pierre, Université Libre de Bruxelles (ULB), Brussels, Belgium. ${ }^{60}$ Pediatric Intensive Care Unit, Robert-Debré University Hospital, APHP, Paris, France. ${ }^{61}$ Sorbonne Paris Nord, Hôpital Jean Verdier, APHP, Bondy, France. ${ }^{62}$ Specialized Immunology Laboratory of Dr. Shahrooei, Sina Medical Complex, Ahvaz, Iran. ${ }^{63}$ Centre de génétique humaine, CHU Besançon, Besançon, France. ${ }^{64}$ Sorbonne Université médecine and APHP Sorbonne université site Pitié-Salpêtrière, Paris, France. ${ }^{65}$ Intensive Care unit, Georges Pompidou Hospital, APHP, Paris, France. ${ }^{66}$ Department of Pneumology, AZ Delta, Roeselare, Belgium. ${ }^{67}$ Institut Jérôme Lejeune, Paris, France. ${ }^{68}$ Department of Microbiology and Immunology, Faculty of Medicine, Mansoura University, Mansoura, Egypt. ${ }^{69}$ Department of Chest, Faculty of Medicine, Mansoura University, Mansoura, Egypt. ${ }^{70}$ Department of Medical Biochemistry and Molecular Biology, Faculty of Medicine, Mansoura University, Mansoura, Egypt. ${ }^{71}$ Faculty of Medicine, Division of Pediatric Infectious Diseases, Selcuk University, Konya, Turkey. ${ }^{72}$ Division of Pediatric Infectious Diseases, Ondokuz Mayıs 
University, Samsun, Turkey. ${ }^{73}$ Necmettin Erbakan University, Meram Medical Faculty, Division of Pediatric Allergy and Immunology, Konya, Turkey. ${ }^{74}$ Centre Hospitalier Fleyriat, Bourg-en-Bresse, France. ${ }^{75}$ Division of Clinical Immunology and Allergy, Department of Internal Medicine, Necmettin Erbakan University, Meram Medical Faculty, Konya, Turkey. ${ }^{76}$ Centre de Génétique, CHU Dijon, Dijon, France. ${ }^{77} \mathrm{APHP}$ Tenon Hospital, Paris, France. ${ }^{78}$ Sorbonne Universités, UPMC University of Paris, Paris, France. ${ }^{79}$ Department of Clinical Immunology , Hospital Clínico San Carlos, Madrid, Spain. ${ }^{80}$ Genomics Division, Instituto Tecnológico y de Energías Renovables (ITER), Santa Cruz de Tenerife, Spain; CIBER de Enfermedades Respiratorias, Instituto de Salud Carlos III, Madrid, Spain; Research Unit, Hospital Universitario N.S. de Candelaria, Santa Cruz de Tenerife, Spain; Instituto de Tecnologías Biomédicas (ITB), Universidad de La Laguna, San Cristóbal de La Laguna, Spain. ${ }^{81} \mathrm{CHU}$ Limoges and Inserm CIC 1435 \& UMR 1092, Limoges, France. ${ }^{82}$ Infectious Diseases Unit, Department of Pediatrics, Hospital Sant Joan de Déu, Barcelona, Spain; Institut de Recerca Sant Joan de Déu, Spain; Universitat de Barcelona (UB), Barcelona, Spain. ${ }^{83}$ Institute of Genetics and Biophysics 'Adriano Buzzati-Traverso', IGB-CNR, Naples, Italy. ${ }^{84}$ Laboratory of Immunogenetics of Human Diseases, IdiPAZ Institute for Health Research, La Paz Hospital, Madrid, Spain. ${ }^{85}$ Hematology, APHP, Hopital Européen Georges Pompidou and Inserm UMR-S1140, Paris, France. ${ }^{86} \mathrm{Hospital}$ General Universitario and Instituto de Investigación Sanitaria "Gregorio Marañón", Madrid, Spain. ${ }^{87}$ Bégin military Hospital, Bégin, France. ${ }^{88}$ Pediatric Intensive Care Unit, Hospital Sant Joan de Déu, Barcelona, Spain. ${ }^{89}$ Department of Internal Medicine, Hôpital Erasme, Université Libre de Bruxelles, Brussels, Belgium. ${ }^{90}$ Division of Critical Care Medicine, Department of Anesthesiology and Reanimation, Necmettin Erbakan University, Meram Medical Faculty, Konya, Turkey. ${ }^{91}$ Genomics Division, Instituto Tecnológico y de Energías Renovables (ITER), Santa Cruz de Tenerife, Spain. ${ }^{92}$ Assistance Publique Hôpitaux de Paris, Paris, France. ${ }^{93}$ Division of Allergy and Immunology, Necmettin Erbakan University, Meram Medical Faculty, Konya, Turkey. ${ }^{94} \mathrm{CNAG}$ CRG, Centre for Genomic Regulation (CRG), Barcelona Institute of Science and Technology (BIST); Universitat Pompeu Fabra (UPF), Barcelona, Spain. ${ }^{95}$ Department of Internal Medicine, National Reference Center for Rare Systemic Autoimmune Diseases, AP-HP, APHP-CUP, Hôpital Cochin, Paris, France. ${ }^{96}$ Ghent University Hospital, Ghent, Belgium. ${ }^{97}$ Sharjah Institute of Medical Research, College of Medicine, University of Sharjah, Sharjah, UAE. ${ }^{98}$ Department of Biosciences and Nutrition, SE14183, Huddinge, Karolinska Institutet, Stockholm, Sweden. ${ }^{99}$ Pediatric Infectious Diseases Unit, Bakirkoy Dr. Sadi Konuk Training and Research Hospital, University of Health Sciences, Istanbul, Turkey. ${ }^{100}$ Department of Immunology , Hospital Universitario de Gran Canaria Dr. Negrín , Canarian Health System, Las Palmas de Gran Canaria, Spain. ${ }^{101}$ Department of Pediatric hemato-oncology, Jolimont Hospital, Belgium; Department of Pediatric hemato-oncology, HUDERF, Brussels, Belgium. ${ }^{102}$ IntensivenCare Unit. Marqués de Valdecilla Hospital, Santander, Spain. ${ }^{103} \mathrm{Hospital}$ del Mar, Parc de Salut Mar, Barcelona, Spain. ${ }^{104}$ Department of Pediatric pulmonology and immunology, Ghent University Hospital, Ghent, Belgium. ${ }^{105}$ Department of Intensive Care Unit, Ghent University Hospital, Ghent, Belgium. ${ }^{106}$ Intensive care unit, APHM, Marseille, France. ${ }^{107} \mathrm{CHU}$ Lille, Lille, France. ${ }^{108}$ Department of Pediatrics, Columbia University , New York, NY, USA. ${ }^{109}$ Centre hospitalier intercommunal Poissy Saint Germain en Laye, Poissy, France. ${ }^{110}$ Division of Respiratory Diseases, Fundació Docència i Recerca Mútua Terrassa, Barcelona, Spain. ${ }^{11}$ Hospital Sant Joan de Déu, Kids Corona Platfform, Barcelona, Spain. ${ }^{112}$ Department of Intensive Care Unit, University Hospital Antwerp, Antwerp, Belgium. ${ }^{113}$ Selcuk University, Faculty of Medicine, Chest Diseases Department, Konya, Turkey. ${ }^{114}$ Division of Allergy and Immunology, Balikesir Ataturk City Hospital, Balikesir, Turkey. ${ }^{115}$ Division of Critical Care Medicine, Selcuk University, Faculty of Medicine, Konya, Turkey. ${ }^{116}$ Division of Pediatric Infectious Diseases, Prof. Dr. Cemil Tascioglu City Hospital, Istanbul, Turkey. ${ }^{117}$ Departments of Infectious Diseases and Clinical Microbiology, Bakirkoy Dr. Sadi Konuk Training and Research Hospital, University of Health Sciences, Istanbul, Turkey. ${ }^{118}$ Meram Medical Faculty, Necmettin Erbakan University, Meram Medical Faculty, Konya, Turkey. ${ }^{119} \mathrm{Health}$ Sciences University, Umraniye Education and Research Hospital, Istanbul, Turkey. ${ }^{120}$ Department of Immunology, 2nd Faculty of Medicine, Charles University and University Hospital in Motol, Prague, Czech Republic. ${ }^{121} \mathrm{Central}$ Clinical Hospital of Ministry of the Interior and Administration in Warsaw, Warsaw, Poland. ${ }^{122}$ Oncobiologie Génétique Bioinformatique, PC Bio, CHU Besançon, Besançon, France. ${ }^{123}$ Paediatric Infectious Disease Unit, Hospital Authority Infectious Disease Center, Princess Margaret Hospital, Hong Kong (Special Administrative Region), China. ${ }^{24}$ Aix Marseille Univ, IRD, MEPHI, IHU Méditerranée Infection, Marseille, France. ${ }^{125}$ Department of Paediatrics and Adolescent Medicine, The University of Hong Kong, Hong Kong, China. ${ }^{126}$ National Centre for Infectious Diseases, Singapore. ${ }^{127}$ Hospital Universitario Reina Sofía, Cordoba, Spain.
${ }^{128}$ Imperial College, London, England. ${ }^{129}$ Endocrinology and diabetes for children, APHP, Bicêtre Paris-saclay hospital , Le Kremlin-Bicêtre, France. ${ }^{130}$ Department of Medical Genetics, University Hospital Antwerp, Antwerp, Belgium. ${ }^{131}$ Neurology unit, APHP Pitié-Salpêtrière Hospital, Paris University, Paris, France. ${ }^{132}$ Intensive care unit, APHP Pitié-Salpêtrière Hospital, Paris University, Paris, France. ${ }^{133}$ National Centre for Infectious Diseases; Tan Tock Seng Hospital; Yong Loo Lin School of Medicine; Lee Kong Chian School of Medicine, Singapore. ${ }^{134}$ Department of Clinical Immunology and Infectious Diseases, National Research Institute of Tuberculosis and Lung Diseases, Shahid Beheshti University of Medical Sciences, , Tehran, Iran. ${ }^{135} \mathrm{Clinical}$ Tuberculosis and Epidemiology Research Center, National Research Institute of Tuberculosis and Lung Diseases (NRITLD), Shahid Beheshti University of Medical Sciences, Tehran, Iran. ${ }^{136}$ Hospital Sant Joan de Déu and University of BarceIona, Barcelona, Spain. ${ }^{137}$ Pediatric Infectious Diseases and Immunodeficiencies Unit, Hospital Universitari Vall d'Hebron, Vall d'Hebron Research Institute, Vall d'Hebron Barcelona Hospital Campus. Universitat Autònoma de Barcelona (UAB), Barcelona, Spain. ${ }^{138}$ Hospital Universitari Mutua de Terrassa, Universitat de BarceIona, Barcelona, Spain. ${ }^{139}$ IrsiCaixa AIDS Research Institute, ICREA, UVic-UCC, Research Institute "Germans Trias i Pujol", Badalona, Spain. ${ }^{140}$ Department of Laboratory, Cruces University Hospital, Barakaldo, Bizkaia, Spain. ${ }^{141}$ University of New South Wales, Australia. ${ }^{142}$ APHP Pitié-Salpêtrière Hospital, Paris, France. ${ }^{143}$ AixMarseille University, APHM, Marseille, France. ${ }^{144}$ Robert Debré Hospital, Paris, France. ${ }^{145} \mathrm{APHP}$ Cohin Hospital, Paris, France. ${ }^{146} \mathrm{Necmettin}$ Erbakan University Meram Faculty of Medicine Department of Pediatric Infectious Diseases, Konya, Turkey. ${ }^{147}$ University Hospitals Leuven, Leuven, Belgium. ${ }^{148} \mathrm{Hospices}$ Civils de Lyon, Hôpital de la Croix-Rousse, Lyon, France. ${ }^{149} \mathrm{Hôpital} \mathrm{Erasme,} \mathrm{Brussels,} \mathrm{Belgium.}{ }^{150} \mathrm{CH}$ gonesse, Gonesse, France. ${ }^{151}$ Vascular Medicine, Georges Pompidou Hospital, APHP, Paris, France. ${ }^{152}$ Division of Pulmonary and Critical Care, University of Miami, Miami, USA. ${ }^{153}$ Guanarteme Health Care Center, Canarian Health System, Las Palmas de Gran Canaria, Spain. ${ }^{154}$ Regional University Hospital of Malaga, Malaga, Spain. ${ }^{155}$ AixMarseille Université, Marseille, France. ${ }^{156}$ Department of General Paediatrics, Hôpital Bicêtre, AP-HP, University of Paris Saclay, Le Kremlin-Bicêtre, France. ${ }^{157}$ Department of Internal Medicine, Ghent University Hospital, Ghent, Belgium. ${ }^{158} \mathrm{CHU}$ de La Timone, Marseille, France. ${ }^{159} \mathrm{Centro}$ Hospitalar Universitário de Lisboa Central, Lisbon, Portugal. ${ }^{160}$ Infectious Diseases Horizontal Technlogy Centre, A*STAR; Singapore Immunology Network, A*STAR, Singapore. ${ }^{161}$ Department of Pediatrics, Complejo Hospitalario Universitario Insular-Materno Infantil, Canarian Health System, Las Palmas de Gran Canaria, Spain. ${ }^{162}$ Regional Universitary Hospital of Malaga, Málaga, Spain. ${ }^{163} \mathrm{H}$ ospital Universitario Marqués de Valdecilla, Santander, Spain. ${ }^{164}$ Faculty of Medicine, Ataturk University, Erzurum, Turkey. ${ }^{165}$ Bilkent University, Department of Molecular Biology and Genetics, Ankara, Turkey. ${ }^{166}$ Department of Laboratory Medicine, Karolinska Institutet, SE14186, Stockholm, Sweden. ${ }^{167}$ L'Hôpital Foch, Suresnes, France. ${ }^{168}$ Department of Immunology, Hospital Universitario ${ }^{12} \mathrm{de}$ Octubre, Instituto de Investigación Sanitaria Hospital 12 de Octubre (imas12), Madrid, Spain. ${ }^{169}$ APHP Hôpitaux Universitaires Paris-Sud, Le Kremlin-Bicêtre, France. ${ }^{170}$ Neurometabolic Diseases Laboratory, IDIBELL-Hospital Duran i Reynals, BarceIona; CIBERER U ${ }^{759}$, ISCiii Madrid, Spain. ${ }^{171}$ Hospices Civils de Lyon, Lyon, France. ${ }^{172}$ Université de Lille, Inserm U1285, CHU Lille, Paris, France. ${ }^{173}$ Departement of General Pediatrics, University Hospital Robert Debré, APHP , Paris, France. ${ }^{174} \mathrm{Necmettin}$ Erbakan University, Konya, Turkey. ${ }^{175}$ Germans Trias i Pujol Hospital, Badalona, Spain. ${ }^{176}$ Medical intensive care unit. Hopital de la Croix-Rousse. Hospices Civils de Lyon, Lyon, France. ${ }^{177}$ Pediatric Infectious Diseases and Immunodeficiencies Unit, Hospital Universitari Vall d'Hebron, Vall d'Hebron Research Institute, Vall d'Hebron Barcelona Hospital Campus., Barcelona, Spain. ${ }^{178}$ Department of Immunology, Hospital Universitario de Gran Canaria Dr. Negrín, Canarian Health System, Las Palmas de Gran Canaria, Spain, EU. University Fernando Pessoa Canarias, Las Palmas de Gran Canaria, Spain. ${ }^{179}$ Neurometabolic Diseases Laboratory, IDIBELL-Hospital Duran i Reynals, Barcelona, Spain. ${ }^{180} \mathrm{Consorcio} \mathrm{Hospital} \mathrm{General} \mathrm{Universitario,} \mathrm{Valen-}$ cia, Spain. ${ }^{181}$ APHP Hôpitaux Universitaires Paris-Sud, Paris, France. ${ }^{182}$ Virology unit, Université de Paris, Cohin Hospital, APHP, Paris, France. ${ }^{183} \mathrm{Hospital}$ San Pedro, Logroño, Spain. ${ }^{184}$ Respiratory medicine, Georges Pompidou Hospital, APHP, Paris, France. ${ }^{185}$ Dept. Immunology, Hospital Clínico San Carlos, Madrid, Spain. ${ }^{186}$ Service de Médecine Intensive Réanimation, Institut de Cardiologie, Hopital Pitié-Salpêtrière. Paris, France. ${ }^{187} \mathrm{CHRU}$ de Nancy, Hôpital d'Enfants, Vandoeuvre, France. ${ }^{188} \mathrm{Chair}$ of Nephrology, University of Brescia, Brescia, Italy. ${ }^{189}$ Department of Immunology, 2nd Faculty of Medicine, Charles University and Motol University Hospital, Prague, Czech Republic. ${ }^{190} \mathrm{Clínica}$ Universidad de Navarra, Madrid, Spain. ${ }^{191}$ HUS Helsinki University Hospital, Children and Adolescents, Rare Disease Center, and Inflammation Center, Adult Immunodeficiency Unit, Majakka, Helsinki, Finland. ${ }^{192}$ Fundació Docència i 
Recerca Mútua Terrassa, Terrassa, Spain. ${ }^{193}$ Department of Pulmonology, ZNA Middelheim, Antwerp, Belgium. ${ }^{194}$ Hopital Européen Georges Pompidou, Paris, France. ${ }^{195}$ Department of Pediatrics, Faculty of Medicine, Mansoura University, Mansoura, Egypt. ${ }^{196} \mathrm{Critical}$ Care Unit , Hospital Universitario de Gran Canaria Dr. Negrín, Canarian Health System, Las Palmas de Gran Canaria, Spain. ${ }^{197} \mathrm{CHU}$ de Saint Etienne, Saint-Priest-en-Jarez, France. ${ }^{198}$ Shupyk National Medical Academy for Postgraduate Education, Kiev, Ukraine. ${ }^{199}$ Gustave Roussy Cancer Campus, Villejuif, France. ${ }^{200}$ Intensive Care Unit, Avicenne Hospital, APHP, Bobigny, France. ${ }^{201}$ Laboratory of Immunology and Histocompatibility, Saint-Louis Hospital, Paris University, Paris, France. ${ }^{202}$ Department of Internal Diseases and Pediatrics, Primary Immune Deficiency Research Lab, Centre for Primary Immunodeficiency Ghent, Jeffrey Modell Diagnosis and Research Centre, Ghent University Hospital, Ghent, Belgium. ${ }^{203} \mathrm{De}-$ partment of Internal Medicine, Université de Paris, INSERM, U970, PARCC, F-75015, Paris, France. ${ }^{204}$ First Division of Anesthesiology and Critical Care Medicine, University of Brescia, ASST Spedali Civili di Brescia, Brescia, Italy. ${ }^{205}$ Intensive Care Department, Hospital Universitari MutuaTerrassa, Universitat Barcelona, Terrassa, Spain. ${ }^{206}$ Hospices Civils de Lyon, Lyon Sud Hospital, Lyon, France. ${ }^{207}$ Infanta Leonor University Hospital, Madrid, Spain. ${ }^{208} \mathrm{Hematology}$ Department, ASST Spedali Civili di Brescia, Brescia, Italy. ${ }^{209}$ Pneumologie, Hôpital Avicenne, APHP, INSERM U1272, Université Sorbonne Paris Nord, Bobigny, France. ${ }^{210}$ Dermatology unit, Laboratoire GAD, INSERM UMR1231 LNC, université de Bourgogne, Dijon, France. ${ }^{211}$ University Hospital of Burgos, Burgos, Spain. ${ }^{212}$ Department of Intensive Care Unit, M. Middelares Ghent, Ghent, Belgium. ${ }^{213}$ Department of Nephrology and Infectiology, AZ Sint-Jan, Bruges, Belgium. ${ }^{214} \mathrm{Center}$ of Human Genetics, Hôpital Erasme, Université Libre de Bruxelles, Brussels, Belgium. ${ }^{215}$ Department of Chest Diseases, Necmettin Erbakan University, Meram Medical Faculty, Konya, Turkey. ${ }^{216} \mathrm{CHU}$ de Caen, Caen, France. ${ }^{217}$ Sorbonne Université, Service de Médecine Intensive Réanimation, Hôpital Tenon, Assistance Publique-Hôpitaux de Paris, Paris, France. ${ }^{218}$ General Intensive Care Unit, Konya Training and Research Hospital, Konya, Turkey. ${ }^{219} \mathrm{CHU}$ de Nancy, Nancy, France. ${ }^{220}$ University of Lyon, CIRI, INSERM U1111, National referee centre RAISE, Pediatric Rheumatology, HFME, Hospices Civils de Lyon, Lyon, France.

*Leader of COVID Clinicians.

\section{Imagine COVID Group}

Christine Bole-Feysot, Stanislas Lyonnet* , Cécile Masson, Patrick Nitschke, Aurore Pouliet, Yoann Schmitt, Frederic Tores, Mohammed Zarhrate

Imagine Institute, Université de Paris, INSERM UMR 1163, Paris, France.

* Leader of the Imagine COVID Group.

\section{French COVID Cohort Study Group}

Laurent Abel ${ }^{1}$, Claire Andrejak ${ }^{2}$, François Angoulvant ${ }^{3}$, Delphine Bachelett, Romain Basmaci $^{5}$, Sylvie Behillii ${ }^{6}$, Marine Beluze ${ }^{7}$, Dehbia Benkerrou ${ }^{8}$, krishna Bhavsar ${ }^{4}$, François Bompart ${ }^{9}$, Lila Bouadma ${ }^{4}$, Maude Bouscambert ${ }^{10}$, Mireille Caralp ${ }^{11}$, Minerva Cervantes-Gonzalez ${ }^{12}$, Anissa Chair ${ }^{4}$, Alexandra Coelho ${ }^{13}$, Camille Couffignal ${ }^{4}$, Sandrine Couffin-Cardiergues ${ }^{14}$, Eric D'ortenzio ${ }^{12}$, Charlene Da Silveira ${ }^{4}$, Marie-Pierre Debray $^{4}$, Dominique Deplanque ${ }^{15}$, Diane Descamps ${ }^{16}$, Mathilde Desvallées ${ }^{17}$, Alpha Diallo ${ }^{18}$, Alphonsine Diouf ${ }^{13}$, Céline Dorival ${ }^{8}$, François Dubos ${ }^{19}$, Xavier Duval $^{4}$, Philippine Eloy $^{4}$, Vincent VE Enouf ${ }^{20}$, Hélène Esperou ${ }^{21}$, Marina Esposito-Farese ${ }^{4}$, Manuel Etienne ${ }^{22}$, Nadia Ettalhaoui ${ }^{4}$, Nathalie Gault ${ }^{4}$, Alexandre Gaymard ${ }^{10}$, Jade Ghosn ${ }^{4}$, Tristan Gigante ${ }^{23}$, Isabelle Gorenne ${ }^{4}$, Jérémie Guedj ${ }^{24}$, Alexandre Hoctin ${ }^{13}$, Isabelle Hoffmann ${ }^{4}$, Salma Jaafoura ${ }^{21}$, Ouifiya Kafif ${ }^{4}$, Florentia Kaguelidou ${ }^{25}$, Sabina Kali ${ }^{4}$, Antoine Khalii ${ }^{4}$, Coralie Khan ${ }^{17}$, Cédric Laouénan ${ }^{4}$, Samira Laribi ${ }^{4}$, Minh Le $e^{4}$, Quentin Le Hingrat ${ }^{4}$, Soizic Le Mestre ${ }^{18}$, Hervé Le Nagard ${ }^{24}$, François-Xavier Lescure ${ }^{4}$, Yves Lévy $^{26}$. Claire Levy-Marchal ${ }^{27}$, Bruno Lina ${ }^{10}$, Guillaume Lingas ${ }^{24}$, Jean Christophe Lucet $^{4}$, Denis Malvy ${ }^{28}$, Marina Mambert ${ }^{13}$, France Mentrée ${ }^{4}$, Noémie Mercier ${ }^{18}$, Amina Meziane ${ }^{8}$, Hugo Mouquet ${ }^{20}$, Jimmy Mullaert ${ }^{4}$, Nadège Neant ${ }^{24}$, Marion Noret ${ }^{29}$, Justine Pages ${ }^{30}$, Aurélie Papadopoulos ${ }^{21}$, Christelle Paul ${ }^{18}$, Nathan Peiffer-Smadja ${ }^{4}$, Ventzislava Petrov-Sanchez ${ }^{18}$, Gilles Peytavin ${ }^{4}$, Olivier Picone ${ }^{31}$, Oriane Puéchal ${ }^{12}$, Manuel Rosa-Calatrava ${ }^{10}$, Bénédicte Rossigno ${ }^{23}$, Patrick Rossigno ${ }^{32}$, Carine Roy ${ }^{4}$, Marion Schneider ${ }^{4}$, Caroline Semaille ${ }^{12}$, Nassima Si Mohammed ${ }^{4}$, Lysa Tagherset ${ }^{4}$, Coralie Tardivon ${ }^{4}$, Marie-Capucine Tellier ${ }^{4}$, François Téoulé ${ }^{8}$, Olivier Terrier ${ }^{10}$, JeanFrançois Timsit ${ }^{4}$, Théo Treoux ${ }^{4}$, Christelle Tua ${ }^{33}$, Sarah Tubiana ${ }^{4}$, Sylvie van der Wer $^{34}$, Noémie Vane ${ }^{35}$, Aurélie Veislinge ${ }^{33}$, Benoit Visseaux ${ }^{16}$, Aurélie Wiedemann ${ }^{26}$, Yazdan Yazdanpanah ${ }^{36}$
${ }^{1}$ Inserm UMR 1163, Paris, France. ${ }^{2} \mathrm{CHU}$ Amiens, France. ${ }^{3}$ Hôpital Necker, Paris, France. ${ }^{4}$ Hôpital Bichat, Paris, France. ${ }^{5}$ Hôpital Louis Mourrier, Colombes, France. ${ }^{6}$ Institut Pasteur, Paris, France. ${ }^{7}$ F-CRIN Partners Platform, AP-HP, Université de Paris, Paris, France. ${ }^{8}$ Inserm UMR 1136, Paris, France. ${ }^{9}$ Drugs for Neglected Diseases initiative, Geneva, Switzerland. ${ }^{10}$ Inserm UMR 1111, Lyon, France. ${ }^{11}$ Inserm Transfert, Paris, France. ${ }^{12}$ REACTing, Paris, France. ${ }^{13}$ Inserm UMR 1018, Paris, France. ${ }^{14}$ Inserm, Pôle Recherche Clinique, France. ${ }^{15} \mathrm{CIC} 1403$ Inserm-CHU Lille, Paris, France. ${ }^{16}$ Université de Paris, IAME, INSERM UMR 1137, AP-HP, University hospital Bichat Claude Bernard, Virology, F-75018 Paris, France. ${ }^{17}$ Inserm UMR 1219, Bordeaux, France. ${ }^{18}$ ANRS, Paris, France. ${ }^{19} \mathrm{CHU}$ Lille, France. ${ }^{20}$ Pasteur Institute, Paris, France. ${ }^{21}$ nnserm sponsor, Paris, France. ${ }^{22}$ Rouen - SMIT, France. ${ }^{23}$ FCRIN INI-CRCT, Nancy, France. ${ }^{24}$ Inserm UMR 1137, Paris, France. ${ }^{25}$ Centre d'Investigation Clinique, Inserm CIC1426, Hôpital Robert Debré, Paris, France. ${ }^{26}$ Inserm UMR 955, Créteil, France; Vaccine Research Instiute (VRI), Paris, France. ${ }^{27} \mathrm{~F}$-CRIN INI-CRCT, Paris, France. ${ }^{28}$ BordeauxSMIT, France. ${ }^{29} \mathrm{RENARCI}$, Annecy, France. ${ }^{30} \mathrm{Hôp}$ ital Robert Debré, Paris, France. ${ }^{31}$ Colombes - Louis Mourier - Gynécologie, France. ${ }^{32}$ University of Lorraine, Plurithematic Clinical Investigation Centre Inserm CIC-P; 1433, Inserm U1116, CHRU Nancy Hopitaux de Brabois, F-CRIN INI-CRCT; (Cardiovascular and Renal Clinical Trialists), Nancy, France. ${ }^{33}$ Inserm CIC-1414, Rennes, France. ${ }^{34}$ Institut Pasteur, UMR 3569 CNRS, Université de Paris, Paris, France. ${ }^{35}$ hôpital la timone, Marseille, France. ${ }^{36}$ Paris - Bichat - SMIT, France.

\section{CoV-Contact Cohort}

Loubna Alavoine ${ }^{1}$, Karine KA Amat² , Sylvie Behillii ${ }^{3}$, Julia Bielicki ${ }^{4}$, Patricia Bruijning ${ }^{5}$, Charles Burdet ${ }^{6}$, Eric Caumes ${ }^{7}$, Charlotte Charpentier ${ }^{8}$, Bruno Coignard ${ }^{9}$, Yolande Costa ${ }^{1}$, Sandrine Couffin-Cardièrgues ${ }^{10}$, Florence Damond ${ }^{8}$, Aline Dechanet ${ }^{11}$, Christelle Delmas ${ }^{10}$, Diane Descamps ${ }^{8}$, Xavier Duvall, Jean-Luc Ecobichon ${ }^{1}$, Vincent Enouf ${ }^{3}$, Hélène Espérou ${ }^{10}$, Wahiba Frezouls ${ }^{1}$, Nadhira Houhou ${ }^{11}$, Emila llic-Habensus ${ }^{1}$, Ouifiya Kafif ${ }^{11}$, John Kikoine ${ }^{11}$, Quentin Le Hingrat ${ }^{8}$, David Lebeaux ${ }^{12}$, Anne Leclercq ${ }^{1}$, Jonathan Lehacaut ${ }^{1}$, Sophie Letrou ${ }^{1}$, Bruno Lina ${ }^{13}$, Jean-Christophe Lucet ${ }^{14}$, Denis Malvy ${ }^{15}$, Pauline Manchon ${ }^{11}$, Milica Mandic ${ }^{1}$, Mohamed Meghadecha ${ }^{16}$, Justina Motiejunaite ${ }^{17}$, Mariama Nouroudine ${ }^{1}$, Valentine Piquard ${ }^{11}$, Andreea Postolache ${ }^{11}$, Caroline Quintin ${ }^{1}$, Jade Rexach ${ }^{1}$, Layidé Roufai ${ }^{10}$, Zaven Terzian ${ }^{11}$, Michael Thy ${ }^{18}$, Sarah Tubiana ${ }^{1}$, Sylvie van der Werf ${ }^{3}$, Valérie Vignali', Benoit Visseaux ${ }^{8}$, Yazdan Yazdanpanah ${ }^{14}$

${ }^{1}$ Centre d'Investigation Clinique, Inserm CIC 1425, Hôpital Bichat Claude Bernard, APHP, Paris, France. ${ }^{2} I M E A$ Fondation Léon M'Ba, Paris, France. ${ }^{3}$ Institut Pasteur, UMR 3569 CNRS, Universite de Paris, Paris, France. ${ }^{4}$ University of Basel Children's Hospital. ${ }^{5}$ Julius Center for Health Sciences and Primary Care, Utrecht. ${ }^{6}$ Université de Paris, IAME, Inserm UMR 1137, F-75018, Paris, France, Hôpital Bichat Claude Bernard, APHP, Paris, France. ${ }^{7}$ Hôpital Pitiè Salpétriere, APHP, Paris. ${ }^{8}$ Université de Paris, IAME, INSERM UMR 1137, AP-HP, University hospital Bichat Claude Bernard, Virology, F-75018 Paris, France. ' ${ }^{9}$ Santé Publique France, Saint Maurice, France. ${ }^{10}$ Pole Recherche Clinique, Inserm, Paris France. ${ }^{11} \mathrm{Hôp}$ ital Bichat Claude Bernard, APHP, Paris, France. ${ }^{12}$ APHP, Paris, France. ${ }^{13}$ Virpath Laboratory, International Center of Research in Infectiology, Lyon University, INSERM U1111, CNRS UMR 5308, ENS, UCBL, Lyon, France. . ${ }^{14}$ IAME Inserm UMR 1138, Hôpital Bichat Claude Bernard, APHP, Paris, France. ${ }^{15}$ Service des Maladies Infectieuses et Tropicales; Groupe Pellegrin-Place Amélie-Raba-Léon, BORDEAUX. ${ }^{16}$ Hôpital Hotel Dieu, APHP, Paris, France. ${ }_{17}^{17}$ ervice des explorations fonctionnelles, Hôpital Bichat-Claude Bernard, APHP, Paris, France. ${ }^{18} \mathrm{Center}$ for Clinical Investigation, Assistance Publique-Hôpitaux de Paris, Bichat-Claude Bernard University Hospital.

\section{Amsterdam UMC Covid-19 Biobank}

Michiel van Agtmael ${ }^{1}$, Anne Geke Algera², Frank van Baarle², Diane Bax³ ${ }^{3}$ Martijn Beudel $^{4}$, Harm Jan Bogaard ${ }^{5}$, Marije Bomers ${ }^{1}$, Lieuwe Bos ${ }^{2}$, Michela Botta², Justin de Brabander ${ }^{6}$, Godelieve Bree ${ }^{6}$, Matthijs C. Brouwer ${ }^{4}$, Sanne de Bruin², Marianna Bugiani ${ }^{7}$ Esther Bulle², O. Chouchane ${ }^{1}$, Alex Cloherty ${ }^{3}$, Paul Elbers², Lucas Fleuren², Suzanne Geerlings ${ }^{1}$, Bart Geerts ${ }^{8}$, Theo Geijtenbeek ${ }^{9}$, Armand Girbes ${ }^{2}$, Bram Goorhuis ${ }^{1}$, Martin P. Grobusch ${ }^{1}$, Florianne Hafkamp ${ }^{9}$, Laura Hagens ${ }^{2}$, Jorg Hamann ${ }^{10}$. Vanessa Harris', Robert Hemke ${ }^{11}$, Sabine M. Hermans ${ }^{1}$, Leo Heunks'2, Markus Hollmann ${ }^{8}$, Janneke Horn², Joppe W. Hovius ${ }^{1}$, Menno de Jong ${ }^{12}$, Rutger Koning ${ }^{4}$, Mourik van Mourik², Jeaninne Nellen ${ }^{1}$, Frederique Paulus'2, Edgar Peters ${ }^{1}$, Tom van der Poll1, Bennedikt Preckel ${ }^{8}$, Jan M. Prins ${ }^{1}$, Jorinde Raasveld ${ }^{2}$, Tom Reijnders ${ }^{1}$, Michiel Schinkel ${ }^{1}$, Marcus Schultz ${ }^{2}$, Alex Schuurman ${ }^{13}$, Kim Sigaloff', Marry Smit' ${ }^{2}$, Cornelis S. Stijnis ${ }^{1}$, Willemke Stilma², Charlotte Teunissen ${ }^{14}$, Patrick Thoral ${ }^{2}$, Anissa Tsonas ${ }^{2}$, Marc van der Valk², Denise Veelo ${ }^{8}$, Alexander P.J. Vlaar ${ }^{15}$, Heder de Vries², Michèle 
van Vugt ${ }^{1}$, W. Joost Wiersinga ${ }^{1}$, Dorien Wouters ${ }^{16}, A . H\left(\right.$ Koos) Zwinderman ${ }^{17}$, Diederik van de Beek ${ }^{18 *}$

1Department of Infectious Diseases, Amsterdam UMC, Amsterdam, Netherlands, ${ }^{2}$ Department of Intensive Care, Amsterdam UMC, Amsterdam, Netherlands. ${ }^{3}$ Experimental Immunology, Amsterdam UMC, Amsterdam, Netherlands. ${ }^{4}$ Department of Neurology, Amsterdam UMC, Amsterdam, Netherlands. ${ }^{5}$ Department of Pulmonology, Amsterdam UMC, Amsterdam, Netherlands. ${ }^{6}$ Department of Infectious Diseases, Amsterdam UMC, Amsterdam, Netherlands. ${ }^{7}$ Department of Pathology, Amsterdam UMC, Amsterdam, Netherlands. ${ }^{8}$ Department of Anesthesiology, Amsterdam UMC, Amsterdam, Netherlands. ${ }^{9}$ Department of Experimental Immunology, Amsterdam UMC, Amsterdam, Netherlands. ${ }^{10}$ Amsterdam UMC Biobank Core Facility, Amsterdam UMC, Amsterdam, Netherlands. ${ }^{11}$ Department of Radiology, Amsterdam UMC, Amsterdam, Netherlands. ${ }^{12}$ Department of Medical Microbiology, Amsterdam UMC, Amsterdam, Netherlands. ${ }^{13}$ Department of Internal Medicine, Amsterdam UMC, Amsterdam, Netherlands. ${ }^{14}$ Neurochemical Laboratory, Amsterdam UMC, Amsterdam, Netherlands. ${ }^{15}$ Department of Intensive Care, Amsterdam UMC, Amsterdam, Netherlands. ${ }^{16}$ Department of Clinical Chemistry, Amsterdam UMC, Amsterdam, Netherlands. ${ }^{17}$ Department of Clinical Epidemiology, Biostatistics and Bioinformatics, Amsterdam UMC, Amsterdam, Netherlands. ${ }^{18}$ Department of Neurology, Amsterdam UMC, Amsterdam, Netherlands.

*Leader of the AMC Consortium.

\section{COVID Human Genetic Effort}

Laurent Abel ${ }^{1}$, Alessandro Aiuti², Saleh Al Muhsen ${ }^{3}$, Fahd Al-Mulla4, Mark S. Anderson $^{5}$, Andrés Augusto Arias ${ }^{6}$, Hagit Baris Feldman ${ }^{7}$, Dusan Bogunovic ${ }^{8}$, Alexandre Bolze ${ }^{9}$, Anastasiia Bondarenko ${ }^{10}$, Ahmed A. Bousfiha ${ }^{11}$, Petter Brodin ${ }^{12}$, Yenan Bryceson $^{12}$, Carlos D. Bustamante ${ }^{13}$, Manish Butte ${ }^{14}$, Giorgio Casari ${ }^{15}$, Samya Chakravorty $^{16}$, John Christodoulou ${ }^{17}$, Elizabeth Cirulli' ${ }^{9}$, Antonio Condino Neto ${ }^{18}$, Megan A. Cooper $^{19}$, Clifton L. Dalgard ${ }^{20}$, AlessiaDavid ${ }^{21}$, Joseph L. DeRisi ${ }^{22}$, Murkesh Desai ${ }^{23}$, Beth A. Drolet ${ }^{24}$, Sara Espinosa ${ }^{25}$, Jacques Fellay ${ }^{26}$, Carlos Flores ${ }^{27}$, Jose Luis Franco ${ }^{28}$, Peter K. Gregersen ${ }^{29}$, Filomeen Haerynck ${ }^{30}$, David Hagin ${ }^{31}$, Rabih Halwani ${ }^{32}$, Jim Heath ${ }^{33}$, Sarah E. Henrickson ${ }^{34}$, Elena Hsieh ${ }^{35}$, Kohsuke Imaij ${ }^{36}$, Yuval Itan ${ }^{8}$, Timokratis Karamitros ${ }^{37}$, Kai Kisand ${ }^{38}$, Cheng-Lung Ku ${ }^{39}$, Yu-Lung Lau ${ }^{40}$, Yun Ling ${ }^{41}$, Carrie L. Lucas ${ }^{42}$, Tom Maniatis ${ }^{43}$, Davoud Mansouri44 ${ }^{44}$, Laszlo Marodi ${ }^{45}$, Isabelle Meyts $^{46}$, Joshua Milner ${ }^{47}$, Kristina Mironska ${ }^{48}$, Trine Mogensen ${ }^{49}$, Tomohiro Morio ${ }^{50}$, Lisa P. Ng ${ }^{51}$, Luigi D. Notarangelo ${ }^{52}$, AntonioNovelli ${ }^{53}$, Giuseppe Novelli ${ }^{54}$, Cliona O'Farrelly ${ }^{55}$, Satoshi Okada ${ }^{56}$, Tayfun Ozcelik ${ }^{57}$, Rebeca Perez de Diego ${ }^{58}$, Anna M. Planas ${ }^{59}$. Carolina Prando ${ }^{60}$, Aurora Pujol ${ }^{61}$, Lluis Quintana-Murci ${ }^{62}$, LaurentRenia ${ }^{63}$, Alessandra Renier ${ }^{64}$, Carlos Rodríguez-Gallego ${ }^{65}$, Vanessa Sancho-Shimizu ${ }^{66}$, Vijay Sankaran ${ }^{67}$, Kelly Schiabor Barrett ${ }^{9}$, Mohammed Shahrooei ${ }^{68}$, Andrew Snow ${ }^{69}$, Pere Soler-Palacín ${ }^{70}$, András N. Spaan ${ }^{71}$, Stuart Tangye ${ }^{72}$, Stuart Turvey ${ }^{73}$, Furkan Uddin ${ }^{74}$, Mohammed J. Uddin ${ }^{75}$. Diederik van de Beek ${ }^{76}$, Sara E. Vazquez ${ }^{77}$, Donald C. Vinh ${ }^{78}$, Horst von Bernuth ${ }^{79}$, Nicole Washington ${ }^{9}$, Pawel Zawadzki ${ }^{80}$, Helen C. Su ${ }^{52}$, Jean-Laurent Casanova ${ }^{81}$

${ }^{1}$ INSERM U1163, University of Paris, Imagine Institute, Paris, France. ${ }^{2}$ San Raffaele Telethon Institute for Gene Therapy, IRCCS Ospedale San Raffaele, Milan, Italy. ${ }^{3}$ King Saud University, Riyadh, Saudi Arabia. ${ }^{4}$ Kuwait University, Kuwait City, Kuwait. ${ }^{5}$ University of California, San Francisco, San Francisco, CA, USA. 'Universidad de Antioquia, Group of Primary Immunodeficiencies, Antioquia, Colombia. ${ }^{7}$ The Genetics Institute, Tel Aviv Sourasky Medical Center and Sackler Faculty of Medicine, Tel Aviv University, Tel Aviv, Israel. ${ }^{8}$ Icahn School of Medicine at Mount Sinai, New York, NY, USA. ${ }^{~} \mathrm{Helix}$, San Mateo, CA, USA. ${ }^{10}$ Shupyk National Medical Academy for Postgraduate Education, Kiev, Ukraine. ${ }^{11} \mathrm{Clinical}$ immunology unit, pediatric infectious disease departement, Faculty of Medicine and Pharmacy, Averroes University Hospital. LICIA Laboratoire d'immunologie clinique, d'inflammation et d'allergie, Hassann li University., Casablanca, Morocco. ${ }^{12}$ Karolinska Institute, Stockholm, Sweden. ${ }^{13}$ Stanford University, Stanford, CA, USA. ${ }^{14}$ University of California, Los Angeles, CA, USA. ${ }^{15}$ Medical Genetics, IRCCS Ospedale San Raffaele, Milan, Italy. ${ }^{16}$ Emory, Atlanta, GA, USA. ${ }^{17}$ Murdoch Children's Research Institute, Victoria, Australia. ${ }^{18}$ University of São Paulo, São Paulo, Brazil. ${ }^{19}$ Washington University School of Medicine, St. Louis, MO, USA. ${ }^{20}$ The American Genome Center; Uniformed Services University of the Health Sciences, Bethesda, MD, USA. ${ }^{21}$ Centre for Bioinformatics and System Biology, Department of Life Sciences, Imperial College London, South Kensington campus, London, UK. ${ }^{2}$ University of California San Francisco; Chan Zuckerberg Biohub, San Francisco, CA, United States. ${ }^{23}$ Bai Jerbai Wadia Hospital for Children, Mumbai, India. ${ }^{24}$ School of Medicine and Public Health, University of Wisconsin, Madison, WI,
USA. ${ }^{25}$ Instituto Nacional de Pediatria (National Institute of Pediatrics), Mexico City, Mexico. ${ }^{26}$ Swiss Federal Institute of Technology Lausanne, Lausanne, Switzerland. ${ }^{27}$ Research Unit, Hospital Universitario Nuestra Señora de Candelaria, Canarian Health System, Santa Cruz de Tenerife, Spain. ${ }^{28}$ University of Antioquia, Medellín, Colombia. ${ }^{29}$ Feinstein Institute for Medical Research, Northwell Health USA, Manhasset, NY, USA. ${ }^{30}$ Department of Paediatric Immunology and Pulmonology, Centre for Primary Immunodeficiency Ghent (CPIG), PID research lab, Jeffrey Modell Diagnosis and Research Centre, Ghent University Hospital, Edegem, Belgium. ${ }^{31}$ The Genetics Institute Tel Aviv Sourasky Medical Center, Tel Aviv, Israel. ${ }^{32}$ Sharjah Institute of Medical Research, College of Medicine, University of Sharjah, Sharjah, UAE. ${ }^{33}$ Institute for Systems Biology, Seattle, WA, USA. ${ }^{34}$ Children's Hospital of Philadelphia, Philadelphia, PA, USA. ${ }^{35}$ Anschutz Medical Campus, Aurora, CO, USA. ${ }^{36}$ Riken, Tokyo, Japan. ${ }^{37}$ Hellenic Pasteur Institute, Athens, Greece. ${ }^{38}$ University of Tartu, Tartu, Estonia. ${ }^{39} \mathrm{Chang}$ Gung University, Taoyuan County, Taiwan. ${ }^{40}$ The University of Hong Kong, Hong Kong, China. ${ }^{41}$ Shanghai Public Health Clinical Center, Fudan University, Shanghai, China. ${ }^{42}$ Yale School of Medicine, New Haven, CT, USA. ${ }^{43}$ New York Genome Center, New York, NY, USA. ${ }^{44}$ Shahid Beheshti University of Medical Sciences, Tehran, Iran. ${ }^{45}$ Semmelweis University Budapest, Budapest, Hungary. ${ }^{46} \mathrm{KU}$ Leuven, Department of Immunology, Microbiology and Transplantation, Leuven, Belgium. ${ }^{47}$ Columbia University Medical Center, New York, NY, USA. ${ }^{48}$ University Clinic for Children's Diseases, Skopje, North Macedonia. ${ }^{49}$ Aarhus University, Aarhus, Denmark. ${ }^{50}$ Tokyo Medical \& Dental University Hospital, Tokyo, Japan. ${ }^{51}$ Singapore Immunology Network, , Singapore. ${ }^{52}$ National Institute of Allergy and Infectious Diseases, National Institutes of Health, Bethesda, MD, USA. ${ }^{53}$ Bambino Gesù Children's Hospital, Rome, Italy, Rome, Italy, Italy. ${ }^{54}$ Bambino Gesù Children's Hospital, Rome, Italy; Dept. Biomedicine and Prevention, University of Rome "Tor Vergata", Rome, Italy. ${ }^{55}$ Trinity College, Dublin, Ireland. ${ }^{56}$ Hiroshima University, Hiroshima, Japan. ${ }^{57}$ Bilkent University, Ankara, Turkey. ${ }^{58}$ Laboratory of Immunogenetics of Human Diseases, Innate Immunity Group, IdiPAZ Institute for Health Research, La Paz Hospital, Madrid 28046, Spain, EU, Madrid, Spain, Spain. ${ }^{59}$ ||BB-CSIC, IDIBAPS, BarceIona, Spain. ${ }^{60}$ Faculdades Pequeno Príncipe e Instituto de Pesquisa Pelé Pequeno Príncipe, Curitiba, Brazil. ${ }^{61}$ Neurometabolic Diseases Laboratory, IDIBELL- Hospital Duran I Reynals; Catalan Institution for Research and Advanced Studies (ICREA); CIBERER U759, ISCiii Madrid Spain, Barcelona, Spain. ${ }^{62}$ Institut Pasteur (CNRS UMR2000) and Collège de France, Paris, France. ${ }^{63}$ nfectious Diseases Horizontal Technology Center and Singapore Immunology Network. Agency for Science Technology (A*STAR), Singapore, Singapore. ${ }^{64}$ University of Siena, Siena, Italy. ${ }^{65}$ Hospital Universitario de Gran Canaria Dr Negrín, Canarian Health System, Canary Islands, Spain. ${ }^{66}$ Imperial College London, London, UK. ${ }^{67}$ Boston Children's Hospital, Harvard Medical School, Boston, MA, USA. ${ }^{68}$ Saeed Pathobiology and Genetic Lab, Tehran, Iran. ${ }^{69}$ Uniformed Services University of the Health Sciences (USUHS), Bethesda, MD, USA. ${ }^{70} \mathrm{Hospital}$ Universitari Vall d'Hebron, Barcelona, Spain. ${ }^{71}$ University Medical Center Utrecht, Amsterdam, The Netherlands. ${ }^{72}$ Garvan Institute of Medical Research, Sydney, Australia. ${ }^{73}$ The University of British Columbia, Vancouver, Canada. ${ }^{74} \mathrm{Holy}$ Family Red Crescent Medical College; Centre for Precision Therapeutics, NeuroGen Children's Healthcare; Genetics and Genomic Medicine Centre, NeuroGen Children's Healthcare, Dhaka, Bangladesh. ${ }^{75}$ Mohammed Bin Rashid University of Medicine and Health Sciences, College of Medicine, Dubai, UAE; The Centre for Applied Genomics, Department of Genetics and Genome Biology, The Hospital for Sick Children, Toronto, Ontario, Canada, Dhaka, Bangladesh. ${ }^{76}$ Amsterdam UMC, Amsterdam, The Netherlands. ${ }^{77}$ University of California, San Francisco, San Francisco, CA, United States. ${ }^{78}$ McGill University Health Centre, Montreal, Canada. ${ }^{79} \mathrm{Charité} \mathrm{-} \mathrm{Berlin}$ University Hospital Center, Berlin, Germany. ${ }^{80}$ Molecular Biophysics Division, Faculty of Physics, A. Mickiewicz University, Uniwersytetu Poznanskiego 2, Poznań, Poland. ${ }^{81}$ Rockefeller University, Howard Hughes Medical Institute, Necker Hospital, New York, NY, USA.

\section{* Leaders of the COVID Human Genetic Effort.}

\section{NIAID-USUHS/TAGC COVID Immunity Group}

Huie Jing ${ }^{1,2}$, Wesley Tung ${ }^{1,2}$, Christopher R. Luthers ${ }^{3}$, Bradly M. Bauman ${ }^{3}$, Samantha Shafer2, ${ }^{2,}$, Lixin Zheng ${ }^{2,4}$, Zinan Zhang ${ }^{2,4}$, Satoshi Kubo 2,4 , Samuel D. Chauvin ${ }^{2,4}$, Kazuyuki Meguro ${ }^{1,2}$, Elana Shaw ${ }^{1.2}$, Michael Lenardo 2,4, Justin Lack ${ }^{5}$, Eric Karlins ${ }^{6}$, Daniel M. Hupelo ${ }^{7}$, John Rosenberger ${ }^{7}$, Gauthaman Sukumar ${ }^{7}$, Matthew D. Wilkerson $^{7}$, Xijun Zhang ${ }^{7}$

${ }^{1}$ Laboratory of Clinical Immunology and Microbiology, Division of Intramural Research, NIAID, NIH, Bethesda, MD, USA. ${ }^{2}$ NIAID Clinical Genomics Program, National Institutes of Health, Bethesda, MD, USA. ${ }^{3}$ Department of Pharmacology \& Molecular 
Therapeutics, Uniformed Services University of the Health Sciences, Bethesda, MD, USA. ${ }^{4}$ Laboratory of Immune System Biology, Division of Intramural Research, NIAID, NIH, Bethesda, MD, USA. ${ }^{5}$ NIAID Collaborative Bioinformatics Resource, Frederick National Laboratory for Cancer Research, Leidos Biomedical Research, Inc. Frederick, MD, USA. ${ }^{6}$ Bioinformatics and Computational Biosciences Branch, Office of Cyber Infrastructure and Computational Biology, NIAID, NIH, Bethesda, MD, USA. ${ }^{7}$ The American Genome Center, Uniformed Services University of the Health Sciences, Bethesda, MD, USA.

\section{SUPPLEMENTARY MATERIALS}

science.sciencemag.org/cgi/content/full/science.abd4570/DC1

Materials and Methods

Figs. S1 to S11

Tables S1 and S2

References (41 and 42)

MDAR Reproducibility Checklist

22 June 2020; accepted 16 September 2020

Published online 24 September 2020

10.1126/science.abd4570 


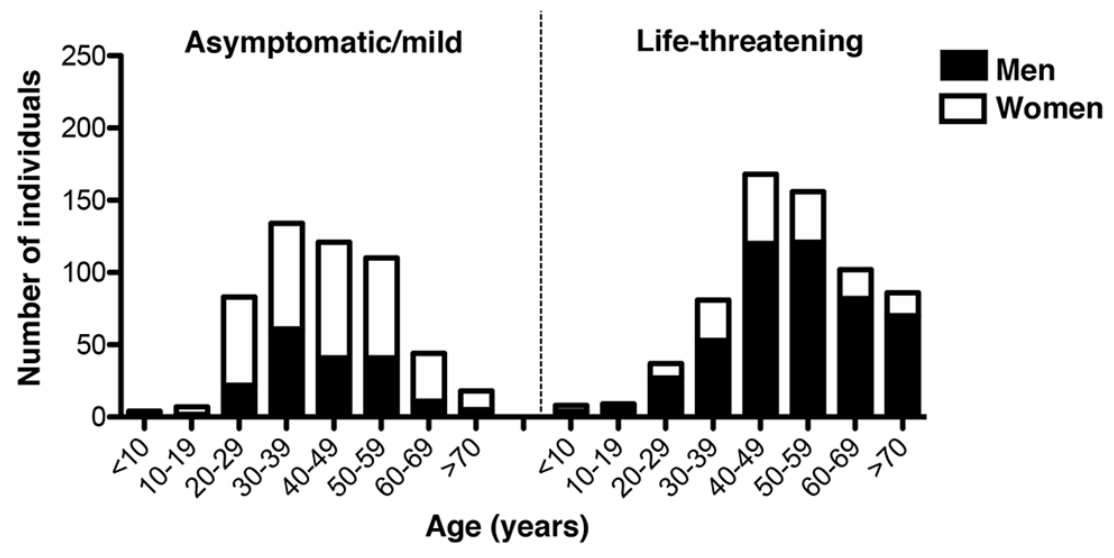

B

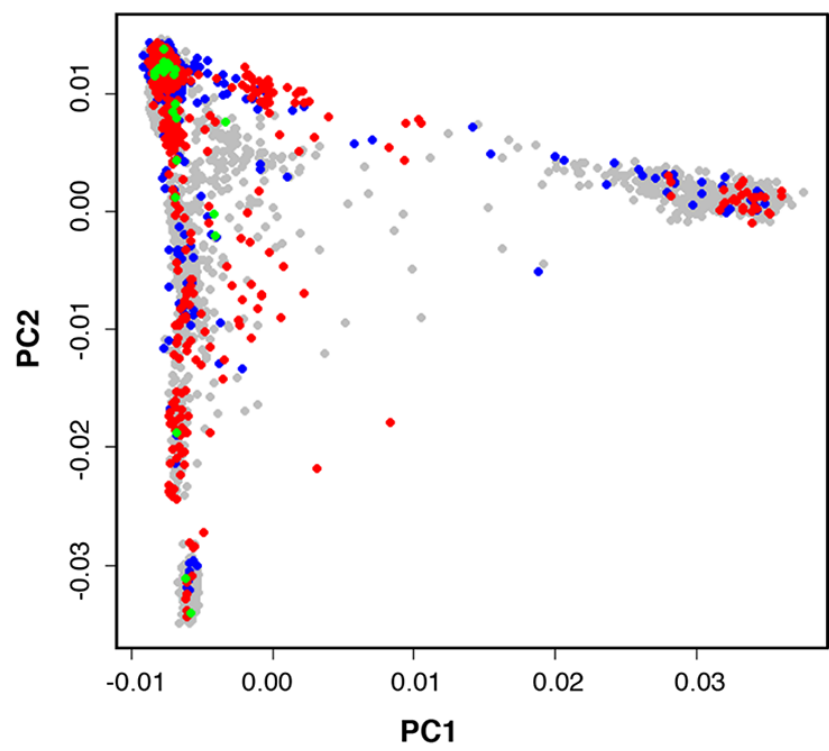

1000 genomes

Asymptomatic/mild COVID

Life-threatening COVID without LOF variants

Life-threatening COVID with LOF variants

Fig. 1. Demographic and genetic data for the COVID-19 cohort. (A) Age and sex distribution of patients with life-threatening COVID-19. (B) PCA of patient and control cohorts (patients with mild/asymptomatic disease and individuals from the 1000 Genomes Project). 


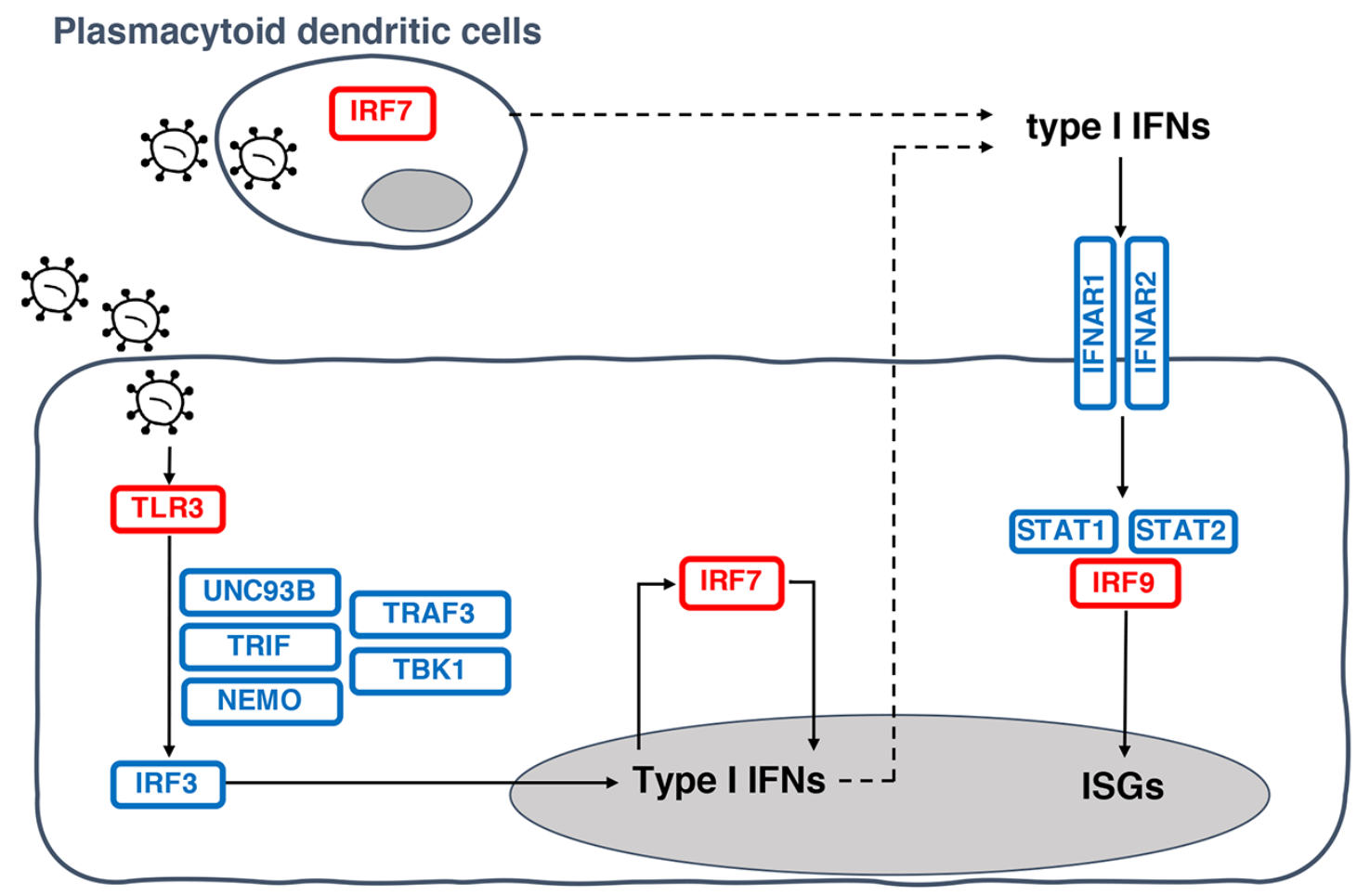

Respiratory epithelial cells

Fig. 2. Illustration of TLR3- and IRF7-dependent type I IFN production and amplification pathway. Molecules in red are encoded by core genes, deleterious variants of which underlie critical influenza pneumonia, with incomplete penetrance, while deleterious variants of genes encoding biochemically related molecules in blue underlie other viral illnesses. ISGs: interferon-stimulated genes. 

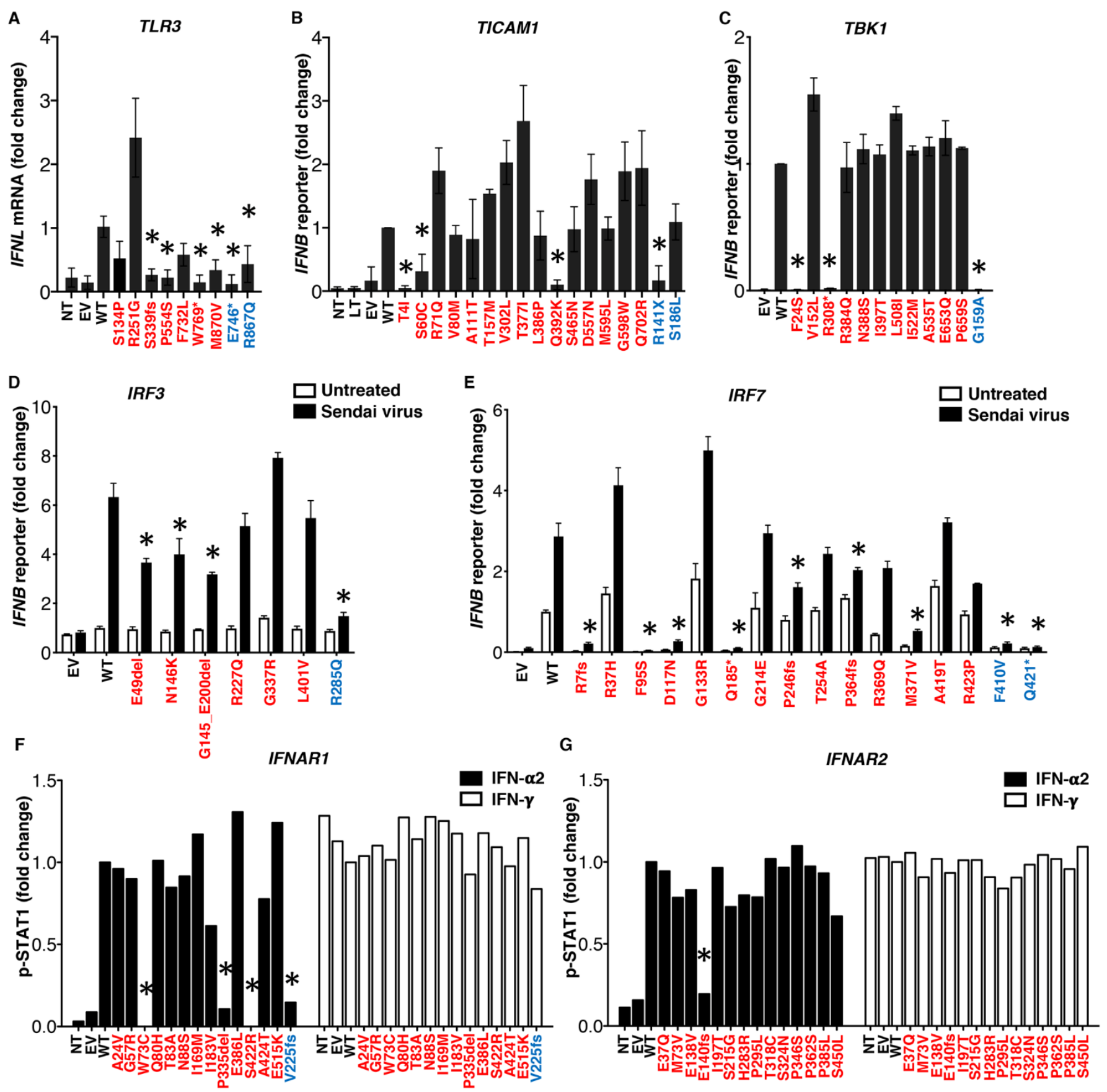
Fig. 3. Impact of TLR3, TICAM1, TBK1, IRF3, IRF7, IFNAR1, and IFNAR2 variants on type I IFN signaling. (A) TLR3deficient P2.1 fibrosarcoma cells were stably transfected with plasmids expressing wild-type or mutant forms of TLR3 and IFNL1 mRNA levels were determined by RT-qPCR. IFNL1 mRNA levels are expressed relative to housekeeping gene GUS, and then normalized. IFNL1 was undetectable in unstimulated cells. The differences between variants and wild-type were tested in one-way ANOVA $\left({ }^{*} p<0.05\right)$. (B) TICAM1-deficient SV40-Fib was transiently transfected with wild-type or mutant forms of TICAM1, together with an IFN- $\beta$-luciferase reporter and a constitutively expressed reporter. Normalized luciferase induction was measured 24 hours after transfection. The differences between variants and wild-type were tested in one-way ANOVA $\left({ }^{*} p<0.05\right)$. (C) HEK293T cells were transiently transfected with wildtype and mutant forms of TBK1, together with an IFN- $\beta$-luciferase reporter and a constitutively expressed reporter. Normalized luciferase activity was measured 24 hours after transfection. The differences between variants and wild-type were tested in one-way ANOVA ( $\left.{ }^{*} p<0.05\right)$. (D) IRF3-deficient HEK293T cells were transiently transfected with wild-type and mutant forms of IRF3, together with an IFN- $\beta$-luciferase reporter and a constitutively expressed reporter. Cells were either left untreated or infected with Sendai virus for 24 hours, before the normalized measurement of luciferase activity. The differences between variants and wild-type were evaluated in two-way ANOVA $\left({ }^{*} p<0.05\right)$. (E) HEK293T cells were transiently transfected with wild-type and mutant forms of IRF7, together with an IFN- $\beta$-luciferase reporter and a constitutively expressed reporter. Cells were either left untreated or infected with Sendai virus for 24 hours before the normalized measurement of luciferase activity. The differences between variants and wild-type were tested in two-way ANOVA $\left({ }^{*} p<0.05\right)$. (F) and $(G)$ IFNAR1- or IFNAR2-deficient SV40-Fib cells were transiently transfected with wild-type or mutant forms of IFNAR1 for 36 hours and were either left untreated or stimulated with IFN- $\alpha 2$ or $-\gamma$. FACS staining with anti-p-STAT1 antibody and the zscore of median fluorescence intensity (MFI) were assessed ( ${ }^{*}$ variants with MFI less than $50 \%$ of wild-type). Variants in red were identified in COVID-19 patients. Variants in blue are known deleterious variants and served as negative controls. EV: empty vector; LT: Lipofectamine; WT: wild-type. Three technical repeats were performed for A-E. Mean and standard deviation (SD) were shown in column and horizontal bars when appropriate. 
A

PHA-T cells

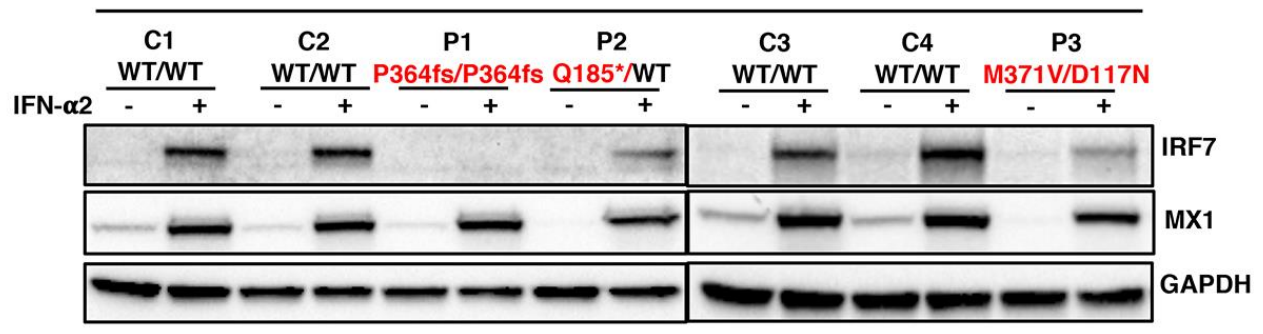

B

pDC
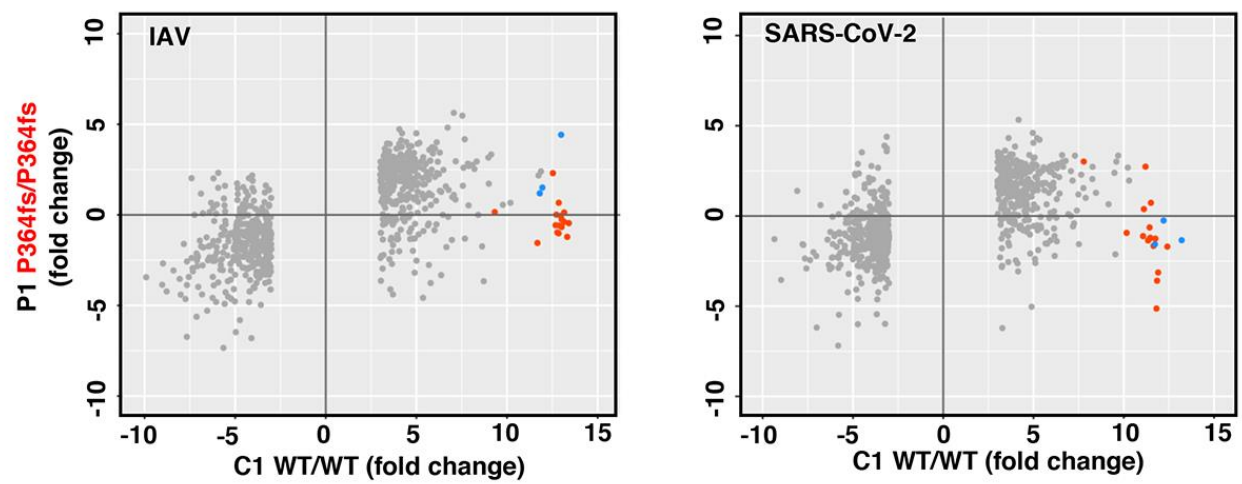

- Type I IFNs

- Type III IFNs

C

pDC
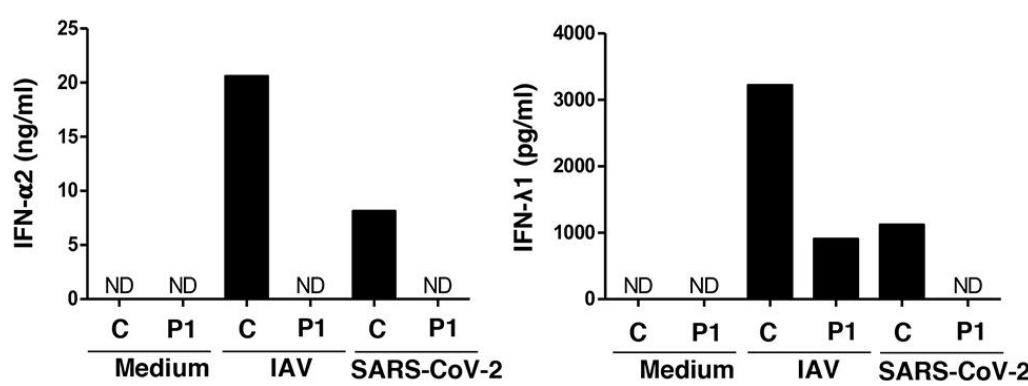

C: IRF7 WT/WT

P1: IRF7 P364fs/P364fs

Fig. 4. Type I IFN responses in patient cells defective for IRF7. (A) Levels of IRF7 protein in PHA-T cells from two patients with AR IRF7 deficiency (P1, P3) and one patient with AD IRF7 deficiency (P2), and four healthy donors (C1-4). Cells were either left untreated or were stimulated with IFN- $\alpha 2$ for 24 hours and protein levels were measured by Western blotting. MX1 was used as a positive control for IFN- $\alpha 2$ treatment. (B) pDCs isolated from an AR IRF7deficient patient (P1) and a healthy donor (C1) were either left untreated, or were infected with influenza A virus (IAV) or SARS-CoV-2, and RNAseq was performed. Genes with expression $>2.5$ fold higher or lower in $\mathrm{C} 1$ after infection are plotted, by fold change in expression. Red dots: type I IFN genes; blue dots: type III IFN genes. (C) pDCs isolated from a healthy donor (C) and IRF7-deficient patient (P1) were either left untreated (Medium) or infected with IAV or SARS-CoV-2, and the production of IFN- $\alpha 2$ and IFN- $\lambda 1$ was measured by CBA and ELISA, respectively, on the supernatant. ND: not detected. 


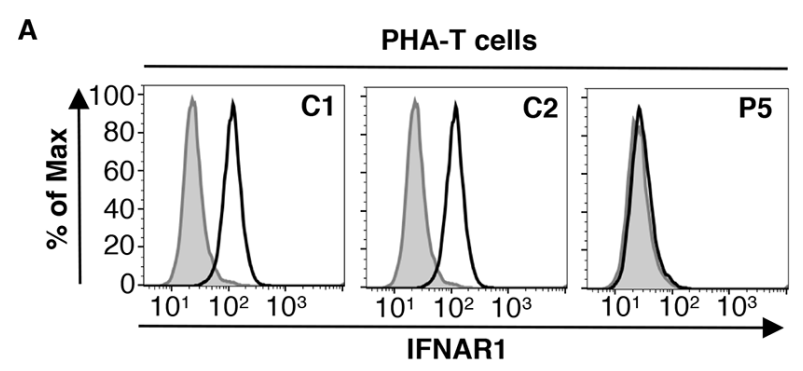

isotype mAb

anti-IFNAR1 mAb

C1, C2: IFNAR1 WT/WT

P5: IFNAR1 W73C/W73C

B

PHA-T cells
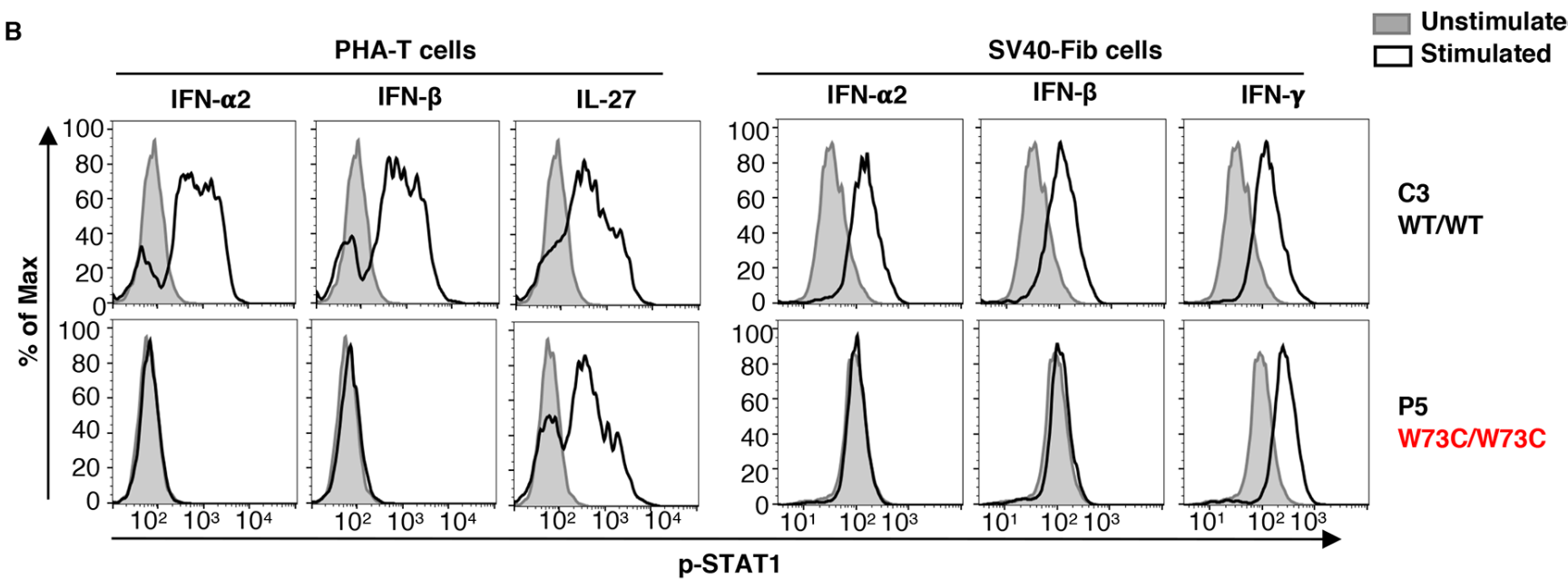

Fig. 5. Type I IFN responses in patient cells defective for IFNAR1. (A) FACS staining of IFNAR1 on the surface of PHA-T cells from a patient with AR IFNAR1 deficiency (P5) and healthy donors (C1, C2). (B) PHA-T cells and SV40-Fib from a patient with AR IFNAR1 deficiency (P5) and a healthy donor (C3) were stimulated with IFN- $\alpha 2$ or $-\beta$, and p-STAT1 levels were determined by FACS. IL-27 stimulation served as a positive control on PHA-T cells, whereas IFN-ץ stimulation served as a positive control on SV40-Fib cells. 


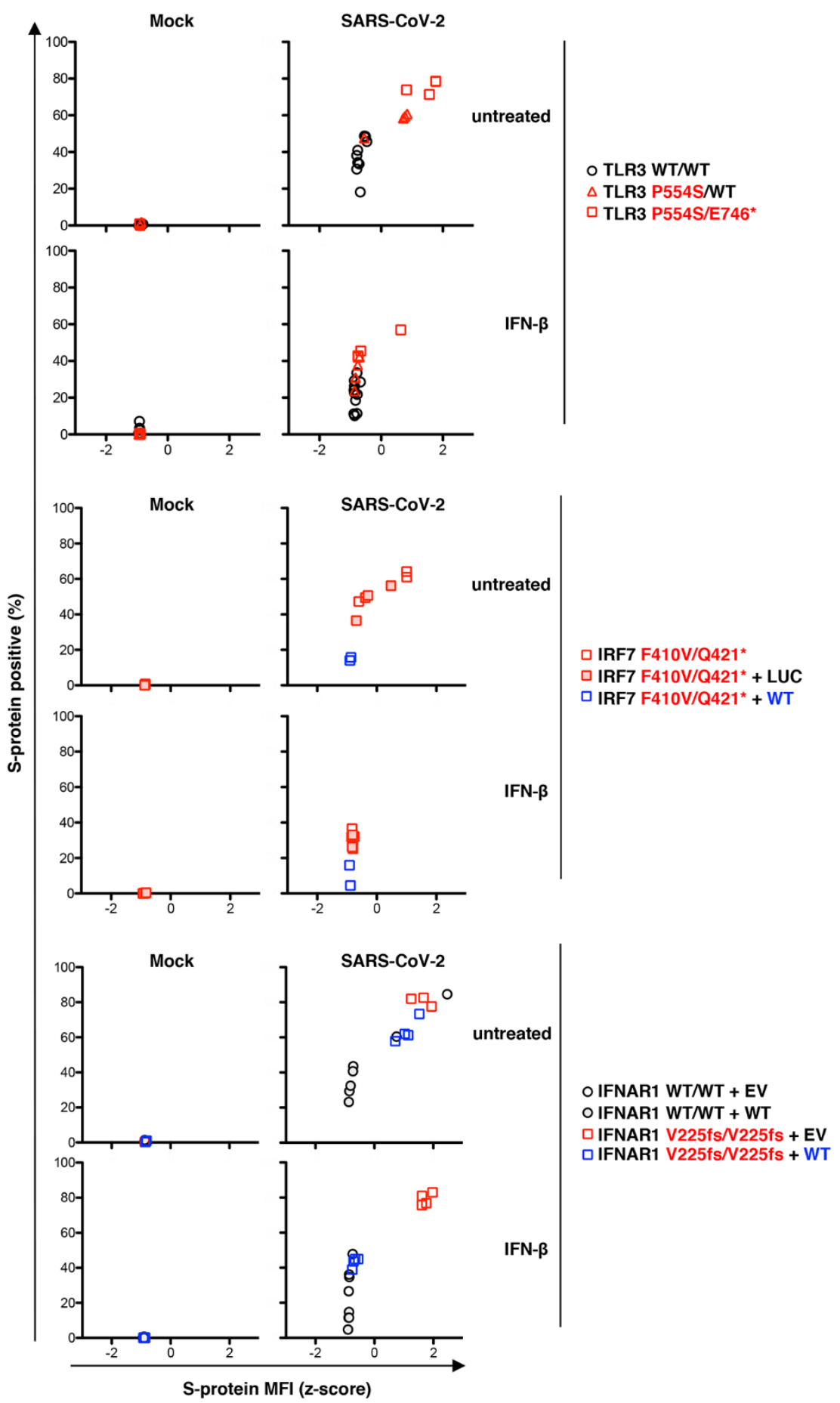

Fig. 6. Cell-intrinsic type I IFN response to SARS-CoV-2. SV40-Fib of TLR3 ${ }^{-/-}, \mathrm{TLR}^{+/-}, \mathrm{IRF}^{-/-}, \mathrm{IRF} 7^{-/-}$rescued with wild-type IRF7, IFNAR1 ${ }^{-1-}$, and IFNAR1 ${ }^{-/-}$rescued with wild-type IFNAR1 were transduced with ACE2 and TMPRSS2 and then either left untreated or treated with IFN- $\beta$ for 4 hours. Cells were then infected with SARSCoV-2 $(\mathrm{MOI}=0.5)$. ACE2 and viral S-protein levels were measured by high-content microscopy, after staining, with gating on ACE2 ${ }^{+}$cells. IRF7 deficient SV40-Fib cells were previously transduced with either wild-type IRF7 (WT IRF7) or negative control (Luc). IFNAR1 deficient cells were previously transduced with either wild-type IFNAR1 (WT IFNAR1) or empty vector (EV). 


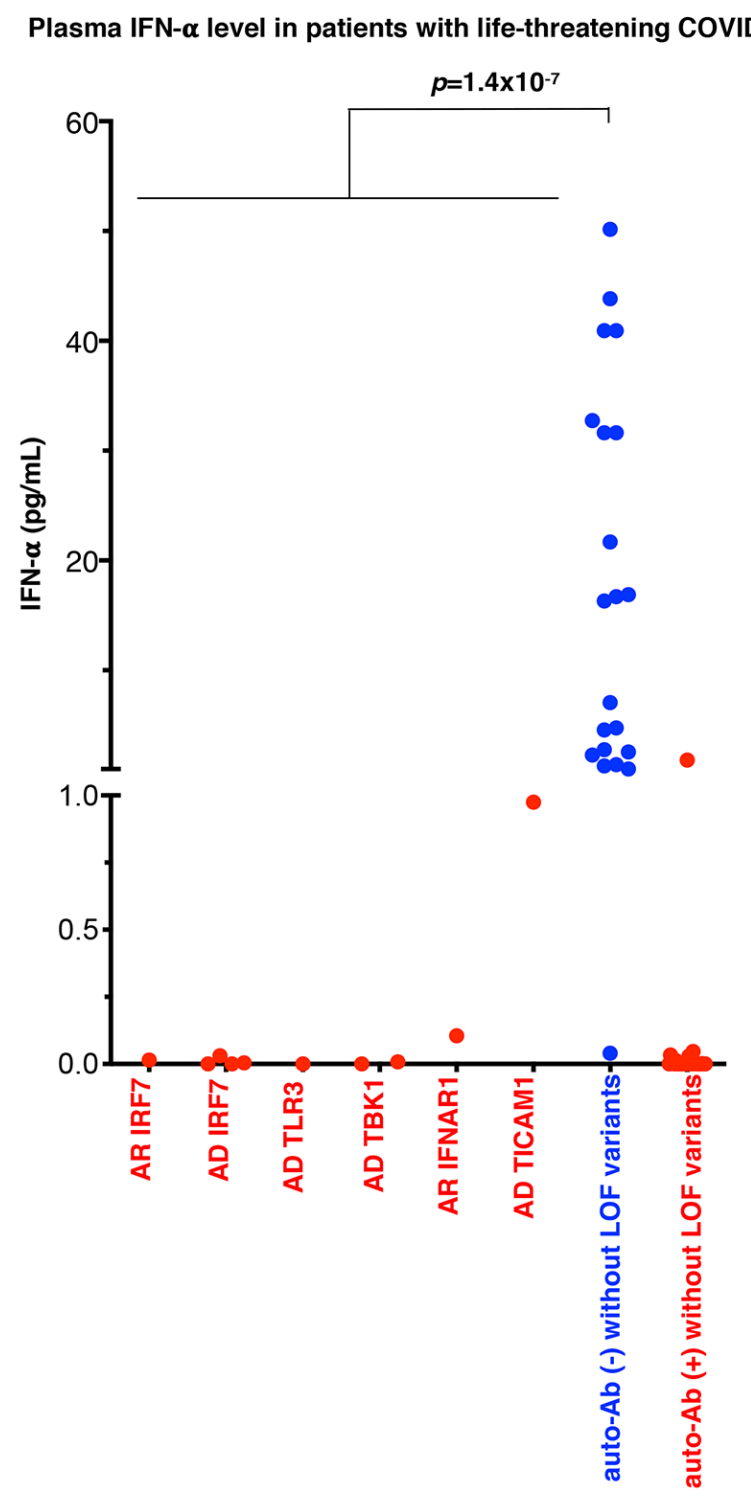

Fig. 7. In vivo type I IFN responses to SARS-CoV-2 infections. Plasma levels of 13 IFN- $\alpha$ were measured by Simoa. AD: autosomal dominant; AR: autosomal recessive; Auto- $\mathrm{Ab}(+)$ without LOF variants: COVID-19 patients with neutralizing anti-IFN- $\alpha$ auto-Abs in our accompanying report (29). $P$ value indicated were evaluated in one-way ANOVA. 
Table 1. Disease-causing variants identified in patients with life-threatening COVID-19.

\begin{tabular}{|c|c|c|c|c|c|c|c|}
\hline Gene & Inheritance & Genetic form & Genotype & Gender & Age (year) & Ancestry/Residence & Outcome \\
\hline TLR3 & $A D$ & Known & p.Ser339fs/WT & M & 40 & Spain & Survived \\
\hline TLR3 & $A D$ & Known & p.Pro554Ser/WT & M & 68 & Italy & Survived \\
\hline TLR3 & $A D$ & Known & p. $\operatorname{Trp} 769 * / \mathrm{WT}$ & M & 77 & Italy & Survived \\
\hline TLR3 & $A D$ & Known & p.Met870Val/WT & M & 56 & Colombian/Spain & Survived \\
\hline UNC93B1 & $A D$ & New & p.Glu96*/WT & M & 48 & Venezuelan/Spain & Survived \\
\hline TICAM1 & $A D$ & Known & p.Thr4lle/WT & M & 49 & Italy & Survived \\
\hline TICAM1 & $A D$ & Known & p.Ser60Cys/WT & $\mathrm{F}$ & 61 & Vietnamese/France & Survived \\
\hline TICAM1 & $A D$ & Known & p.Gln392Lys/WT & $\mathrm{F}$ & 71 & Italy & Deceased \\
\hline TBK1 & $A D$ & Known & p.Phe24Ser/WT & $\mathrm{F}$ & 46 & Venezuelan/Spain & Survived \\
\hline TBK1 & $A D$ & Known & p.Arg308*/WT & M & 17 & Turkey & Survived \\
\hline IRF3 & $A D$ & Known & p.Glu49del/WT & $\mathrm{F}$ & 23 & Bolivian/Spain & Survived \\
\hline IRF3 & $A D$ & Known & p.Asn146Lys/WT & $\mathrm{F}$ & 60 & Italy & Survived \\
\hline IRF7 & AR & Known & p.Pro364fs/p.Pro364fs & $\mathrm{F}$ & 49 & Italian/Belgium & Survived \\
\hline IRF7 & AR & Known & p.Met371Val/p.Asp117Asn & M & 50 & Turkey & Survived \\
\hline IRF7 & $A D$ & New & p.Arg7fs/WT & M & 60 & Italy & Survived \\
\hline IRF7 & $A D$ & New & p.GIn185*/WT & M & 44 & France & Survived \\
\hline IRF7 & $A D$ & New & p.Pro246fs/WT & M & 41 & Spain & Survived \\
\hline IRF7 & $A D$ & New & p.Arg369GIn/WT & M & 69 & Italy & Survived \\
\hline IRF7 & $A D$ & New & p.Phe95Ser/WT & M & 37 & Turkey & Survived \\
\hline IFNAR1 & AR & Known & p.Trp73Cys/Trp73Cys & M & 38 & Turkey & Survived \\
\hline IFNAR1 & AR & Known & p.Ser422Arg/Ser422Arg & M & 26 & Pakistan/Saudi Arabia & Deceased \\
\hline IFNAR1 & $A D$ & New & p.Pro335del/WT & $\mathrm{F}$ & 23 & Chinese/Italy & Survived \\
\hline IFNAR2 & $A D$ & New & p.Glu140fs/WT & $\mathrm{F}$ & 54 & Belgium & Survived \\
\hline
\end{tabular}

AD: autosomal dominant; AR: autosomal recessive; WT: wild-type. 